OPEN ACCESS

Edited by:

Elodie Segura,

Institut Curie, France

Reviewed by:

Geert Van Den Bogaart,

University of Groningen, Netherlands

Bénédicte Manoury,

Institut National de la Santé et de la

Recherche Médicale (INSERM),

France

${ }^{*}$ Correspondence:

Weijuan Yao

weijuanyao@bjmu.edu.cn

Zhu Zeng

zengzhu@gmc.edu.cn

${ }^{\dagger}$ These authors have contributed equally to this work

Specialty section:

This article was submitted to Antigen Presenting Cell Biology,

a section of the journal

Frontiers in Immunology

Received: 26 July 2020 Accepted: 03 December 2020 Published: 15 January 2021

Citation:

Liu X, Xia X, Wang X, Zhou J, Sung LA, Long J, Geng X, Zeng $Z$ and Yao W (2021) Tropomodulin1 Expression

Increases Upon Maturation in Dendritic Cells and Promotes Their Maturation and Immune Functions.

Front. Immunol. 11:587441. doi: $10.3389 /$ fimmu.2020.587441

\section{Tropomodulin1 Expression Increases Upon Maturation in Dendritic Cells and Promotes Their Maturation and Immune Functions}

\author{
Xianmei Liu ${ }^{1,2 \dagger}$, Xue Xia ${ }^{1,2 \dagger}$, Xifu Wang ${ }^{3}$, Jing Zhou ${ }^{2}$, Lanping Amy Sung ${ }^{4}$, \\ Jinhua Long ${ }^{1}$, Xueyu Geng ${ }^{2}$, Zhu Zeng ${ }^{1 *}$ and Weijuan Yao ${ }^{2,5^{*}}$ \\ 1 School of Basic Medical Sciences, School of Biology and Engineering, Guizhou Medical University, Guiyang, China, \\ 2 Hemorheology Center, Department of Physiology and Pathophysiology, School of Basic Medical Sciences, Peking \\ University Health Science Center, Beijing, China, ${ }^{3}$ Department of Emergency, Beijing Anzhen Hospital, Capital Medical \\ University, Beijing, China, ${ }^{4}$ Department of Bioengineering, University of California, San Diego, La Jolla, CA, United States, \\ ${ }^{5}$ Department of Integration of Chinese and Western Medicine, School of Basic Medical Science, Peking University Health \\ Center, Beijing, China
}

Dendritic cells (DCs) are the most potent antigen-presenting cells. Upon maturation, DCs express costimulatory molecules and migrate to the lymph nodes to present antigens to $T$ cells. The actin cytoskeleton plays key roles in multiple aspects of DC functions. However, little is known about the mechanisms and identities of actin-binding proteins that control DC maturation and maturation-associated functional changes. Tropomodulin1 (Tmod1), an actin-capping protein, controls actin depolymerization and nucleation. We found that Tmod1 was expressed in bone marrow-derived immature DCs and was significantly upregulated upon lipopolysaccharide (LPS)-induced DC maturation. By characterizing LPS-induced mature DCs (mDCs) from Tmod1 knockout mice, we found that compared with $\mathrm{Tmod}^{+/+}$mDCs, Tmod1-deficient mDCs exhibited lower surface expression of costimulatory molecules and chemokine receptors and reduced secretion of inflammatory cytokines, suggesting that Tmod1 deficiency retarded DC maturation. Tmod1-deficient mDCs also showed impaired random and chemotactic migration, deteriorated T-cell stimulatory ability, and reduced F-actin content and cell stiffness. Furthermore, Tmod1deficient $\mathrm{mDC}$ s secreted high levels of IFN- $\beta$ and IL-10 and induced immune tolerance in an experimental autoimmune encephalomyelitis (EAE) mouse model. Mechanistically, Tmod1 deficiency affected TLR4 signaling transduction, resulting in the decreased activity of MyD88-dependent NFKB and MAPK pathways but the increased activity of the TRIF/ IRF3 pathway. Rescue with exogenous Tmod1 reversed the effect of Tmod1 deficiency on TLR4 signaling. Therefore, Tmod1 is critical in regulating DC maturation and immune functions by regulating TLR4 signaling and the actin cytoskeleton. Tmod1 may be a potential target for modulating DC functions, a strategy that would be beneficial for immunotherapy for several diseases.

Keywords: dendritic cells, maturation, Tmod1, TLR4 signaling, antigen presentation 


\section{INTRODUCTION}

Dendritic cells (DCs) are the most potent antigen-presenting cells (APCs) and play critical roles in initiating and exaggerating innate and adaptive immune responses $(1,2)$. DC-based immunotherapy against cancer and autoimmune diseases has achieved promising clinical outcomes (3-6). Originating from bone marrow precursors, immature DCs (imDCs) reside in the peripheral non-lymphoid tissues and execute the immune surveillance function (7). Upon capturing antigens through pattern recognition receptors such as Toll-like receptors (TLRs), imDCs gradually develop into mature DCs (mDCs) through the activation of MyD88- and TRIF-dependent signaling pathways $(8,9)$. This occurs in concert with the translocation of MHC-II from endosomal compartments to the plasma membrane, and the upregulation of costimulatory molecules, chemokine receptors, and cytokines (10-13). Simultaneously, mDCs migrate to the lymphoid organs and present the antigenic peptides to naïve $\mathrm{T}$ cells to mediate immune responses $(14,15)$. Although a considerable amount of information about the biological behaviors of DCs is available, the molecular mechanisms underlying their differentiation, maturation, and immune functions of DCs remain elusive.

The Actin cytoskeleton undergoes remodeling during DC maturation, and plays critical roles in regulating the morphology, adhesion, migration, endocytosis, and antigen presentation (1619). As regulators of the actin cytoskeleton, many actin-binding proteins are found differentially expressed during DC maturation and contribute to the maturation-associated functional changes. For example, the actin bundling protein, Fascin1, is upregulated upon maturation and promotes the migration of mDCs (20). The activation of Cofilin is correlated with the morphological changes during DC maturation (21). Members of the Rho family GTPases, Rac1 and Rac2, control the formation of dendrites and their T-cell priming in mDCs (22). Another Rho family GTPase, Cdc42, stops expressing during DC maturation and regulates the secretory activity of mDCs (23). The scaffolding protein, Caveolin-1, is upregulated in DCs upon maturation and promotes their migration to lymph nodes through Rac1dependent actin cytoskeleton remodeling to elicit effective Tcell responses (24). Interestingly, knockout of Fascin1 or Caveolin-1 does not alter the expression of DC maturation markers $(20,24)$. However, Cdc42 knockout results in phenotypically mature DCs, that express high levels of CD86 on the cell surface (23). These findings indicate that actinbinding proteins may play different roles in regulating the maturation process and immune functions of DCs, which needs further studies.

Tropomodulin1 (Tmod1) is an actin-capping protein that was first isolated in human erythrocytes (25). By binding to tropomyosin (TM) and actin, Tmodl caps the pointed end of TM-coated actin filaments and decreases the rate of actin depolymerization (26). Tmod1 also has actin nucleating activity depending on its actin monomer binding and pointed end-capping activity $(27,28)$. Therefore, Tmod 1 plays important roles in regulating actin dynamics and cytoskeleton structure, and further determines cell morphology, cell mechanics, contraction, and dendritic protrusion growing from neurons $(29,30)$. Knockout of Tmod1 in mice results in embryonic lethality because of non-contractile heart tube with disorganized myofibrils, accumulation of mechanically weakened primitive erythroid cells in the yolk sac, and failure of primary capillary plexus remodeling $(31,32)$. X-gal staining of $T_{m o d}{ }^{+/-}$mice with lacZ gene knock-in revealed that Tmod1 was expressed in mouse peripheral blood mononuclear cells (PBMCs) (33). Using microarray and RNA-sequencing techniques, several studies have revealed that Tmod1 is significantly upregulated in PBMCs or macrophages of patients with advanced renal cell carcinoma, inflammatory bowel diseases, or rheumatoid arthritis (34-36). These results indicate that Tmod 1 is expressed in immune cells and is possibly involved in regulating inflammation and immune responses.

In the present study, we demonstrated that Tmod 1 is expressed in bone marrow-derived dendritic cells (BMDCs) and is upregulated upon lipopolysaccharide (LPS)-induced DC maturation. Using BMDCs from TOT/Tmod1 ${ }^{-1-}$ mice, that were obtained by crossing cardiomyocyte-specific Tmod 1 overexpressing transgenic (TOT) mice with $\mathrm{Tmodl}^{+/-}$mice (37, 38), we showed that Tmod1 deficiency retarded DC maturation by downregulating the expression of costimulatory molecules and inflammatory cytokines, and impaired their migration and T-cell stimulatory abilities by altering their actin cytoskeleton and cell mechanics. Furthermore, LPS-treated Tmod1-deficient DCs secreted high levels of IFN- $\beta$ and IL-10, and induced immune tolerance in an experimental autoimmune encephalomyelitis (EAE) mouse model. Mechanistically, Tmod1 deficiency affected TLR4 signaling transduction, resulting in decreased activity of NFKB and MAPK pathways but increased activity of the IRF3 pathway. Rescue with exogenous Tmod1 reversed the effect of Tmod1 deficiency on TLR4 signaling. Therefore, Tmod1 is critical in regulating DC maturation and immune functions, possibly by regulating TLR4 signaling and the actin cytoskeleton.

\section{MATERIALS AND METHODS}

\section{Reagents and Antibodies}

Recombinant murine granulocyte-macrophage colony stimulating factor (rmGM-CSF) and recombinant murine interleukin 4 (rmIL-4) were purchased from Peprotech (Rocky Hill, NJ, USA). LPS was purchased from Sigma (St. Louis, MO, USA). $\beta$-mercaptoethanol was obtained from MP Biomedicals LLC (Solon, OH, USA). Pam3csk4, poly (I:C), and CpG ODN1826 were purchased from InvivoGen (San Diego, CA, USA). FITC-, APC-, and PE-conjugated anti-murine CD4, CD11c, CD40, CCR7, CD80, CD86, and MHC-II antibodies were purchased from BioLegend (San Diego, CA, USA) and eBiosciences (New York, NY, USA). Antibodies against CD11c, TLR4, MyD88, and IאB were purchased from Biosynthesis Biotechnology Co. Ltd. (Beijing, China). Antibodies against p65 and p38 were obtained from Zhongshan Golden Bridge Co. Ltd. (Beijing, China). Antibodies against phospho-p65, phospho-IRF3, phospho-ERK1/2, and IRF3 were from Cell 
Signaling Technology (Danvers, MA, USA). Antibodies against phospho-p38 and ERK1/2 were purchased from Bioworld Technology (Nanjing, China). Anti-Tmod1 antibody was prepared by AbMax Biotechnology Co., Ltd. (Beijing, China). Antibodies against GAPDH and $\beta$-actin were obtained from Santa Cruz Biotech. (Santa Cruz, CA, USA). Tmod1 adenovirus and control adenovirus were constructed by SinoGeneMax Co. Ltd. (Beijing, China).

\section{Animals and Culture of BMDCs}

A Tmod1 knockout mouse model was first created by disrupting exon 1 in Dr. L. Amy Sung's laboratory at the University of California, San Diego (31). While $\operatorname{Tmod1}^{-/-}$mice died at embryonic day 9.5, the heterozygous $\operatorname{Tmod1}^{+/}$mice were able to mate with a cardiac specific Tmod1 overexpressing transgenic (TOT) mice (37). Interbreeding between their offsprings resulted in TOT/Tmod1 $1^{-1-}$ mice where the overexpression of Tmod 1 in the heart rescued the homozygosity's lethality (38). TOT/Tmod1 ${ }^{-/}$ mice were transferred from Dr. L. Amy Sung's lab to the Peking University Health Science Center and maintained in SPF animal room. C57BL/6J $\left(\operatorname{Tmod1}^{+/+}\right)$mice were purchased from the animal department of the Peking University Health Science Center. The animal study was approved by the ethics committee of the Peking University Health Science Center. BMDCs were obtained from 8 week-old female TOT/Tmod1 $1^{-/}$ and $T \bmod 1^{+/+}$mice according to the method developed by Roney (39). Briefly, bone marrow cells were flushed out from freshly dissected femurs and tibias. Following lysis of the red blood cells in lysis buffer, the remaining cells were resuspended in Roswell Park Memorial Institute 1640 (RPMI 1640) medium supplemented with $10 \%$ fetal bovine serum, $1 \%$ penicillinstreptomycin, and $0.1 \% \beta$-mercaptoethanol, and differentiated into imDCs in the presence of $20 \mathrm{ng} / \mathrm{ml} \mathrm{rmGM-CSF}$ and $10 \mathrm{ng} /$ $\mathrm{ml} \mathrm{rmIL-4}$ for 7 days. The imDCs were then matured into mDCs by adding $100 \mathrm{ng} / \mathrm{ml}$ LPS for another 2 days. The imDCs were also treated with Pam3csk4 (100 ng/ml), poly (I:C) $(25 \mu \mathrm{g} / \mathrm{ml})$, and CpG ODN 1826 (1 $\mu \mathrm{M})$, respectively, for 1-2 days.

\section{Flow Cytometry}

ImDCs and mDCs were fixed with $4 \%$ paraformaldehyde and labeled with FITC-, PE-, or APC-conjugated antibodies to mouse CD11c, CD86, MHC-II, CD80, CD40, and CCR7, and the corresponding isotype controls. The cells were then analyzed with a BD FACS Calibur ${ }^{\mathrm{TM}}$ flow cytometer (BD Biosciences, San Diego, CA, USA).

\section{Western Blotting}

ImDCs and mDCs were harvested and lysed in RIPA buffer containing protease inhibitor cocktail and $1 \mathrm{mM}$ phenylmethylsulfonyl fluoride (PMSF). Total proteins $(100 \mu \mathrm{g})$ were separated on a $10 \%$ sodium dodecyl sulfate-polyacrylamide gel electrophoresis (SDS-PAGE) gel and transferred onto a nitrocellulose membrane. After blocking with 5\% nonfat milk, the membrane was incubated with antibodies against Tmod1, GAPDH, CD11c, p65, p-p65, p38, p-p38, ERK1/2, p-ERK1/2, and TLR4 overnight at $4^{\circ} \mathrm{C}$. After washing, the membranes were incubated with HRP-conjugated secondary antibody for $1 \mathrm{~h}$ at room temperature $\left(25^{\circ} \mathrm{C}\right)$. The signals were visualized using an enhanced chemiluminescence (ECL) detection kit (Evergreen, Beijing, China) and exposed to X-ray films.

\section{Real-Time Quantitative PCR}

Total RNA was extracted from imDCs and mDCs using RNAtrip reagent (Applygen Biotechnologies Inc., Beijing, China) and then reverse transcribed into cDNA using the First Strand cDNA Synthesis Kit (Thermo Fisher Scientific, Waltham, MA, USA). Real-time quantitative PCR (RT-qPCR) was performed on a Mx3000 Multiplex Quantitative PCR system (Stratagene, La Jolla, CA, USA) using EvaGreen qPCR Master Mix-low Rox (Applied Biological Materials (abm) Inc., Richmond, BC, Canada). The sequences of the primers are listed in Table 1. GAPDH was used as an internal control. A relative fold change in the gene expression was calculated using the method of $2^{-\triangle \triangle \mathrm{Ct}}$ method.

\section{Adenovirus Infection on Tmod1-Deficient DCs}

About $5 \times 10^{5}$ TOT/Tmod1 $1^{-/}$imDCs were infected with Tmod 1 adenovirus (Ad-Tmod1) or control adenovirus (Ad-Null) at 200 MOI (multiplicity of infection) for $6 \mathrm{~h}$. Equal number of TOT/ $\mathrm{Tmod1}^{-/-}$imDCs without adenovirus treatment were used as the negative control. The cells were further treated with LPS (100 ng/ $\mathrm{mL}$ ) for $24 \mathrm{~h}$ before cells and culture media were collected.

TABLE 1 | The list of primer sequences.

\begin{tabular}{|c|c|}
\hline \multirow[t]{2}{*}{ Tmod1 } & 5'-GACACAGCCTCACACAATGT-3'/ \\
\hline & 5'-CTTGGTGGTCTGATCCTTCT-3' \\
\hline \multirow[t]{2}{*}{ CD80 } & 5'-GGCAAGGCAGCAATACCTTA-3'/ \\
\hline & 5'-CCATGTCCAAGGCTCATTCT-3' \\
\hline \multirow[t]{2}{*}{ CD86 } & 5'-GGCAGATATGCAGTCCCATT-3'/ \\
\hline & 5'-AGAACTTACGGAAGCACCCA-3' \\
\hline \multirow[t]{2}{*}{ CD40 } & 5'-CTGGCACAAATCACAGCACT-3'/ \\
\hline & 5'-CTGCATGGTGTCTाTGCCT-3' \\
\hline \multirow[t]{2}{*}{ CCR7 } & 5'- GAAGGCTGTGCTाTGGTTC-3'/ \\
\hline & 5'-CAAACAGGAGCTGATGTCCA-3' \\
\hline \multirow[t]{2}{*}{ MHC-II } & 5'-ACCGTGTTCTGCTCATCCT-3'/ \\
\hline & 5'-AGTTCTCAAAGTAGTGCCT-3' \\
\hline \multirow[t]{2}{*}{ IL-6 } & 5'-GATGGATGCTACCAAACTGGA-3'/ \\
\hline & 5'-TCTGAAGGACTCTGGCTITG-3' \\
\hline \multirow[t]{2}{*}{ TNF- $\alpha$} & 5'- CAGCCTCTTCTCATTCCTGC-3'/ \\
\hline & 5'-GGTCTGGGCCATAGAACTGA-3' \\
\hline \multirow[t]{2}{*}{$\mathrm{IL}-12 \mathrm{a}$} & 5'- CTAGACAAGGGCATGCTGGT-3'/ \\
\hline & 5'-GCTTCTCCCACAGGAGGTाT-3' \\
\hline \multirow[t]{2}{*}{ IFN- $\gamma$} & 5'-ATGAACGCTACACACTGCATC-3'/ \\
\hline & 5'-CCATCCTITGCCAGTTCCTC-3' \\
\hline \multirow[t]{2}{*}{ IFN- $\beta$} & 5'- AATTTCTCCAGCACTGGGTG-3' \\
\hline & 5'-AGTTGAGGACATCTCCCACG-3' \\
\hline \multirow[t]{2}{*}{$\mid \mathrm{L}-10$} & 5'- TACACCTGCGTाTCTCAGCC-3'/ \\
\hline & 5'-CAGTATTGCACTCTGTAAGCCC-3' \\
\hline \multirow[t]{2}{*}{ TLR4 } & 5'-TGTTCTTCTCCTGCCTGACA-3'/ \\
\hline & 5'-TGTCATCAGGGACTTGCTG-3' \\
\hline \multirow[t]{2}{*}{ Tmod2 } & 5'-TTGGAGAGGGTGAAAGAGAGGG-3'/ \\
\hline & 5'-GTGTTGGTAATGGGATTGGGAT-3' \\
\hline \multirow[t]{2}{*}{ Tmod3 } & 5'-ACTATATCCCTCGACCCAGA-3'/ \\
\hline & 5'-GACACCGTTACTACTTCCCA-3' \\
\hline \multirow[t]{2}{*}{ Tmod4 } & 5'-GTGATGCGGTAGAGATGGAGAT-3'/ \\
\hline & 5'-CTTGTTGGTAAAAGGAAGGGTG-3' \\
\hline \multirow[t]{2}{*}{ GAPDH } & 5' - ACCACAGTCCATGCCATCAC-3'/ \\
\hline & 5'-TCCACCACCCTGTTGCTGTA-3' \\
\hline
\end{tabular}




\section{Measurement of Cyotkines and Chemokines by Multiplex Analysis or Enzyme Linked Immunosorbent Assay}

Culture media of imDCs, mDCs, and OVA peptide-treated spleen cells were collected. The levels of cytokines and chemokines, including interleukin-6 (IL-6), tumor necrosis factor- $\alpha$ (TNF- $\alpha$ ), interleukin-12 (IL-12), interferon- $\gamma$ (IFN- $\gamma$ ), interferon- $\beta$ (IFN- $\beta$ ), and interleukin-10 (IL-10), were measured by flow cytometry using LEGENDplex ${ }^{\text {TM }}$ bead-based immunoassay kits (BioLegend). The levels of TNF- $\alpha$ and IL-10 in the culture media of adenovirus-infected and LPS-treated TOT/Tmod1 $1^{-/}$imDCs and TLR agonist-treated imDCs were measured using Enzyme linked immunosorbent assay (ELISA) kits (BOSTER Biotechnology, Wuhan, China).

\section{Endocytosis Assay for imDCs}

Approximately $5 \times 10^{5}$ imDCs were incubated with $500 \mu \mathrm{g} / \mathrm{ml}$ FITC-dextran particles (43.2 kDa, Sigma) in $1 \mathrm{ml}$ of RPMI 1640 at $4^{\circ} \mathrm{C}$ or $37^{\circ} \mathrm{C}(40)$. After 0.5 or $2 \mathrm{~h}$ of incubation, the cells were washed three times with PBS to remove the un-endocytosed particles and the endocytosed particles were analyzed using flow cytometry. The differences in the fluorescence intensities between $37^{\circ} \mathrm{C}$ and $4^{\circ} \mathrm{C}$ represented the endocytosis abilities of imDCs.

\section{Migration Assay}

Migration of imDCs and mDCs was measured using a modified Boyden chamber (5- $\mu \mathrm{m}$ pore size). A total of $2 \times 10^{5}$ cells in $200 \mu \mathrm{l}$ volume was plated in the upper compartment of the chamber, and $600 \mu \mathrm{l}$ of complete medium supplemented with or without $200 \mathrm{ng}$ CCL19 was added to the lower compartment. The chamber was incubated for $12 \mathrm{~h}$ at $37^{\circ} \mathrm{C}$, following which the migrated cells were counted under a light microscope.

\section{Allogeneic Mixed Lymphocyte Reaction}

Mixed lymphocyte reaction (MLR) assay was used to determine the T-cell activation abilities of the mDCs (40). Allogeneic T cells were obtained from the spleen cells of $\mathrm{C} 57 \mathrm{BL} / 6 \mathrm{~J}$ mice by passing the spleen cells through a nylon wool column. mDCs $\left(1 \times 10^{5}\right.$, $1 \times 10^{4}$, and $1 \times 10^{3}$ ) were mixed with $1 \times 10^{5} \mathrm{~T}$ cells, respectively, in a total volume of $200 \mu \mathrm{l}$, and the mixtures were incubated in a $\mathrm{CO}_{2}$ incubator for $48 \mathrm{~h}$. Then, $20 \mu \mathrm{l}$ CCK8 solution (Enhanced Cell Counting Kit-8, Beyotime Biotech., Shanghai, China) was added to each mixture for $4 \mathrm{~h}$. The proliferation of the $\mathrm{T}$ cells was quantified by measuring the optical density at 450 $\mathrm{nm}$ in a microplate reader (BioRad, Hercules, CA, USA).

\section{Adoptive Transfer With DCs and In Vitro Restimulation of T Cells}

Mature DCs were incubated with $10 \mu \mathrm{g} / \mathrm{ml}$ OVA peptide (323339) (Alpha Diagnostic Intl Inc., San Antonio, TX, USA) at $37^{\circ} \mathrm{C}$ for $2 \mathrm{~h}$. The cells were washed twice and resuspended in serumfree RPMI 1640 medium. Treated mDCs $\left(1 \times 10^{6}\right)$ were injected intravenously into $\mathrm{C} 57 \mathrm{BL} / 6 \mathrm{~J}$ mice on days 1,3 , and 5 . On day 8 , the mice were sacrificed, and their spleen cells were collected, and re-stimulated with $10 \mu \mathrm{g} / \mathrm{ml} \mathrm{OVA} \mathrm{peptide} \mathrm{(323-339)} \mathrm{at} 37^{\circ} \mathrm{C}$ for
3 days (41). Then, the spleen cells were washed with PBS, stained with FITC-conjugated anti-mouse CD4 antibody, and further analyzed by flow cytometry. The fluorescence intensity indicated the proliferation of $\mathrm{CD}^{+} \mathrm{T}$ cells. The concentrations of IFN- $\gamma$ and IL-10 in culture medium of OVA-peptide-treated spleen cells were measured by flow cytometry using LEGENDplex ${ }^{\mathrm{TM}}$ bead-based immunoassay kits (BioLegend).

\section{Preparation of Myelin Oligodendrocyte Glycoprotein (MOG)35-55 Peptide-Pulsed mDCs and Induction of EAE}

Mature DCs were pulsed with $20 \mu \mathrm{g} / \mathrm{ml}$ MOG35-55 peptide (Sigma) for $1 \mathrm{~h}$ at $37^{\circ} \mathrm{C}(41)$. The pulsed cells $\left(1.2 \times 10^{6}\right)$ were injected intravenously into female C57BL/6J mice twice (on days 0 and 4). Experimental autoimmune encephalomyelitis (EAE) was induced by subcutaneous injection of $100 \mu \mathrm{g}$ of MOG35-55 peptide emulsified in complete Freund's adjuvant (Chondrex, Redmond, WA, USA) with $100 \mu \mathrm{l}$ of $4 \mathrm{mg} / \mathrm{ml}$ Mycobacterium tuberculosis (Chondrex) on days 7 and 14. In addition, $200 \mathrm{ng}$ of pertussis toxin (Invitrogen, Carlsbad, CA, USA) was injected intraperitoneally on days 7 and 9. The mice were observed and scored on the scale of 0 to 5 : 0 , no symptoms; 1 , flaccid tail; 2 , hind limb weakness; 3 , partial hind limb paralysis; 4, complete hind limb paralysis; 5, moribund state (41).

\section{Measurement of F-Actin Content in the DCs}

ImDCs and mDCs were fixed in $4 \%$ paraformaldehyde and washed twice with PBS. After permeabilization with $0.1 \%$ Triton X-100, the cells were blocked with $1 \%$ bovine serum albumin (BSA) for $30 \mathrm{~min}$ at room temperature. The cells were then stained with $0.165 \mu \mathrm{M}$ rhodamine phalloidin (Invitrogen) in the dark for $20 \mathrm{~min}$. The cells were washed, resuspended in PBS, and analyzed using a BD FACS Calibur (BD Bioscience). The mean fluorescence intensity represented the F-actin content in the cells.

\section{Laser Scanning Confocal Microscopy}

ImDCs and mDCs were cultured on poly L-lysine-treated coverslips and stained with rhodamine phalloidin as mentioned above. The nuclei were stained with 4',6-diamidino2-phenylindole (DAPI, Beyotime Biotech., Shanghai, China) for 5 min. After washing with PBS, the cells were observed under a laser scanning confocal microscope (Leica TCS SP8 MP FLIM, Wetzlar, Germany).

\section{Measurement of Young's Moduli of the DCs by Nanoindenter}

ImDCs and mDCs were cultured on poly-L-lysine-treated coverslips. The petri dish containing the coverslip was placed on an inverted microscope equipped with a nanoindenter (Piuma Chiaro, Optic II, Amsterdam, Netherlands). A probe with spring constant of $0.18 \mathrm{~N} / \mathrm{m}$ and a spherical tip radius of 9$\mu \mathrm{m}$ was mounted on the cantilever. During indentation, the tip was brought into contact with the cell surface and loadindentation and load-time data were recorded. The indentation 
depth was $10 \mu \mathrm{m}$ and the loading and unloading time was set to $2 \mathrm{~s}$. The loading and unloading curves were fitted with Hertzian contact model and Young's modulus was calculated. The mean Young's modulus for each specimen was generated from at least 20 to 30 cells from three independent preparations.

\section{Statistical Analysis}

All experimental procedures were repeated at least three times and are presented as mean \pm standard error of mean (SEM). GraphPad Prism 7.0 software was used for the normal distribution test, homogeneity of variance test, statistical analysis, and plotting. Paired or unpaired Student's $t$-tests were used to compare the results of the two groups, and analysis of variance was used between the groups. $\mathrm{P}<0.05$ was considered statistically significant.

\section{RESULTS}

\section{Tmod1 Is Expressed in BMDCs and Upregulated Upon LPS-Induced Maturation}

To define the immune functions of Tmod1, we first examined whether Tmod1 was expressed in DCs. We isolated bone marrow cells from $\mathrm{C} 57 \mathrm{BL} / 6 \mathrm{~J}\left(\operatorname{Tmod} 1^{+/+}\right)$mice and differentiated them into imDCs following treatment with IL-4 and GM-CSF. LPS was used to induce DC maturation. qPCR data showed that compared with imDCs, Tmod1 was upregulated by approximately 4 -fold in mDCs (Figure 1A, p < 0.05). Western blotting data further confirmed that Tmod1 was present in imDCs and its expression level increased significantly upon DC maturation (Figure 1B, p $<0.05$ ). We wondered whether agonists for other TLRs could also promote Tmod1 expression. Therefore, imDCs of Tmod1 $^{+/+}$mice were treated with TLR2 agonist Pam3csk4 and TLR3 agonist poly (I:C). Un-treated and LPS-treated imDCs served as a negative and positive control. Both qPCR and western blotting data showed that neither Pam3csk4 nor poly (I:C) could stimulate Tmod1 expression (Figures S1A, B). This suggests that LPS is the strongest stimulation for Tmod1 expression among these TLR agonists.

We also cultured DCs of TOT/Tmod1 ${ }^{-/-}$mice and analyzed Tmod1 expression in these cells. However, no Tmod 1 could be detected, suggesting the complete absence of Tmod1 in TOT/ Tmod1 $1^{-/-}$DCs (Figure 1B). In addition to Tmod1, three homologs of similar size exist in Tmod family, Tmod2 (neuronal specific), Tmod3 (ubiquitous), and Tmod4 (skeletal muscle specific) (42). Compensatory effect is observed among some of the homologs (43). To examine the expression of other Tmod homologs in Tmod1-deficient DCs, we performed qPCR on imDCs and LPS-induced mDCs of Tmod1 $1^{+/+}$and TOT/ $\mathrm{Tmod1}^{-/-}$mice. Results showed that the expression levels of Tmod2, Tmod3, and Tmod4 were all comparable between the two genotypes (Figure S2). This suggests that other Tmod homologs did not compensate for the loss of Tmod1.

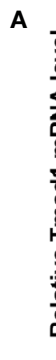

C
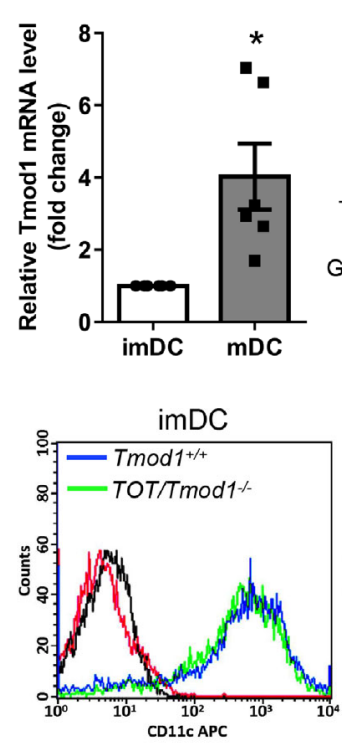

B
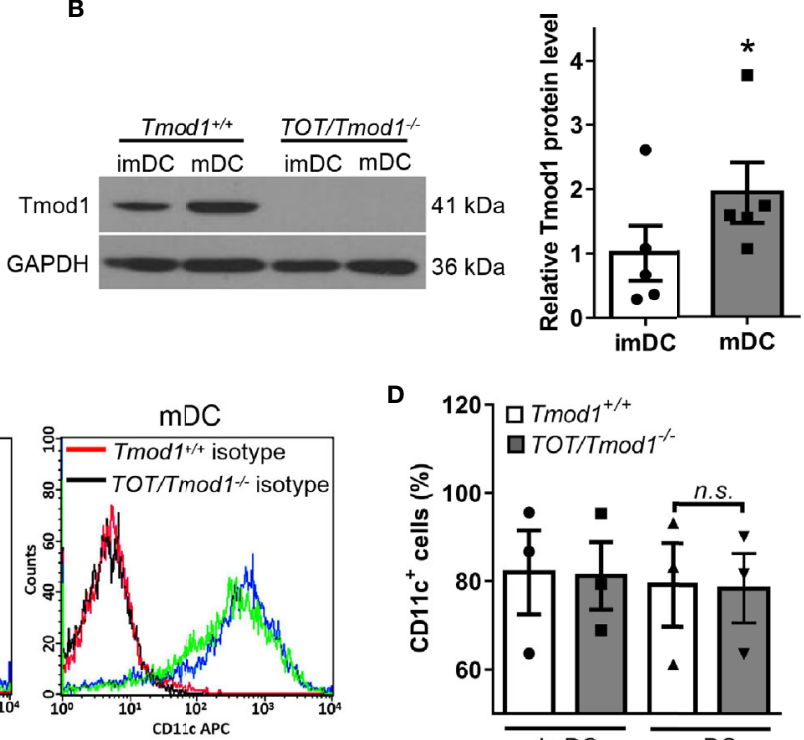

D

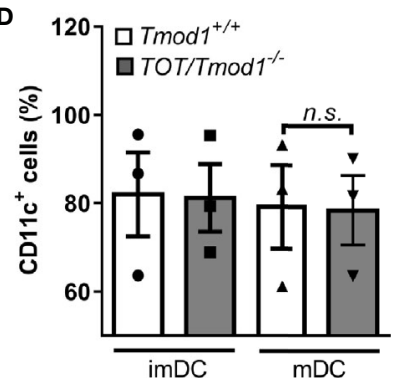

FIGURE 1 | The expression of Tmod1 increased upon maturation in dendritic cells (DCS) induced by lipopolysaccharide (LPS) and Tmod1 deficiency did not influence DC differentiation. (A) Bone marrow-derived immature DCs (imDCs) of wild type (Tmod1 ${ }^{+/+}$) mice were treated with LPS (100 ng/ml) for 2 days to induce the maturation. The mRNA level of Tmod1 in imDCs and mature DCs (mDCs) was detected by qPCR. (B) ImDCs from Tmod $1^{+/+}$and TOT/Tmod1 ${ }^{-/-}$mice were induced to mature DCs (mDCs) with LPS. The protein level of Tmod1 in imDCs and mDCs of both genotypes was detected by western blotting. Tmod1 expression in Tmod $1^{+/+}$imDCs and mDCs was quantified after normalization to GAPDH (right panel). (C) The imDCs and mDCs from Tmod1 ${ }^{+/+}$and TOT/Tmod1 ${ }^{-/}$mice were stained with APC-conjugated antibody for DC marker, CD11c, and analyzed with flow cytometry. The representative flow cytometry histograms were shown. (D) The

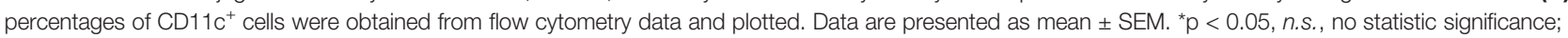
paired, two-tailed student's $t$-test in (A, B), un-paired, two-tailed student's $t$-test in (C). 


\section{Tmod1 Is Not Essential for the Differentiation of BMDCs}

Since Tmod1 is expressed in both imDCs and mDCs, we sought to determine whether Tmod1 plays a role in DC differentiation. We measured the expression level of the DC marker, CD11c, on the surface of imDCs and mDCs from Tmod1 $1^{+/+}$and TOT/ $\mathrm{Tmod1}^{-/-}$mice. Flow cytometry data showed that CD11c levels were comparable in Tmod1 $1^{+/+}$and TOT/Tmod1 $1^{-/-} \mathrm{DCs}$, regardless of their state of maturation (immature or mature; Figures 1C, D). We also analyzed CD11c levels in the cell lysates of Tmod $^{+/+}$and TOT/Tmod1 $1^{-/-}$DCs using western blotting, but did not detect any difference (Figure S3). These results suggest that Tmod1 is not essential for the differentiation of BMDCs.

\section{Tmod1 Is Required for LPS-Induced Maturation and Cytokine Secretion in DCs}

The finding that Tmod1 was upregulated upon maturation suggests that Tmod1 may be involved in the maturation process and the functional changes related to maturation. DC maturation is characterized by increased surface expression of costimulatory molecules and chemokine receptors as well as the secretion of cytokines. Therefore, we examined the expression of MHC-II, CD80, CD86, CD40, and CCR7 on Tmod1-deficient DCs using flow cytometry. The results showed that Tmod1deficient imDCs had considerably lower expression of MHC-II and CCR7 than Tmod $1^{+/+}$imDCs (Figures 2A, B). However, the expression of CD80, CD86, and CD40 was similar between the two groups. Following LPS induction, the expression of MHC-II, CD80, CD86, CD40, and CCR7 increased markedly on both Tmod1 $^{+/+}$and TOT/Tmod1 $1^{-/}$mDCs. However, the expression levels of these molecules were significantly lower on TOT/ Tmod1 $^{-/-}$mDCs than on Tmod1 $1^{+/+}$mDCs (Figures 2A, B), suggesting that TOT/Tmod1 $1^{-/}$mDCs were phenotypically less mature than Tmod1 ${ }^{+/+}$mDCs. We also compared the levels of cytokines secreted by Tmod $1^{+/+}$and TOT/Tmod $1^{-/-}$mDCs. The data showed that compared with $T \bmod 1^{+/+} m \mathrm{mCs}$, TOT/ $\mathrm{Tmod1}^{-1-} m \mathrm{DCs}$ secreted noticeably less TNF- $\alpha$, IFN- $\gamma$, and IL12 (p70) ( $p<0.05$, Figure 2C). IL-6 secretion was similar in both groups. These results indicate that Tmod1 deficiency retarded LPS-induced DC maturation by reducing surface marker expression and cytokine secretion.

\section{Tmod1 Regulates LPS-Induced Phosphorylation of NF-kB and p38 MAPK in DCs}

The decreased expression of multiple surface markers and secretion of several cytokines suggests that Tmod1 deficiency may affect the activities of upstream signaling pathways. TLR4 is the receptor for LPS and its downstream MyD88-dependent pathway activates NF- $\kappa \mathrm{B}$ and MAPK pathways, which in turn are responsible for the expression of costimulatory molecules and inflammatory cytokines $(44,45)$. Therefore, we compared the activities of these two pathways in LPS-treated $T \bmod 1^{+/+}$and TOT/Tmod1 $1^{-/}$imDCs using western blotting. We found that $\mathrm{I} \kappa \mathrm{B}$, which retains $\mathrm{p} 65$ in the cytosol, started to degrade within 5 min of LPS treatment and was almost undetectable by $30-60$ $\min$ in $T \bmod 1^{+/+}$imDCs (Figure 3A). However, in TOT/ Tmod1 $1^{-/}$imDCs, although it started to become degraded within $5 \mathrm{~min}$, it was still present in large amounts (1.7fold relative to $T \bmod 1^{+/+}$) at 30-60 min, suggesting that the degradation of $I \kappa B$ was retarded (Figures $3 \mathbf{A}, \mathbf{B}$ ). Correspondingly, p65 phosphorylation increased in $5 \mathrm{~min}$ and reached the highest level at $30 \mathrm{~min}$ in both $T \bmod 1^{+/+}$and TOT/ Tmod $1^{-/}$imDCs, but the phosphorylation level was considerably lower in TOT/Tmod1 $1^{-/}$imDCs than in Tmod $1^{+/+}$imDCs $(80 \%$ less at $5 \mathrm{~min}$, Figures $\mathbf{3 A}, \mathbf{B})$. Although LPS-induced phosphorylation kinetics of p38 MAPK was similar between Tmod $^{+/+}$and TOT/Tmod1 $1^{-/-}$imDCs, its phosphorylation level in TOT/Tmod1 $1^{-/}$imDCs was considerably lower than that in Tmod $^{+/+}$imDCs $(30 \%$ less at $5 \mathrm{~min}$, Figures $3 \mathrm{~A}, \mathbf{B})$. No difference was found in the phosphorylation levels of ERK1/2 MAPK between the two groups (Figure S4). Our results indicate that in the absence of Tmod1, LPS-induced phosphorylation of the key signaling molecules, $\mathrm{NF}-\kappa \mathrm{B}$ and p38 MAPK, is significantly compromised.

Next, we examined the mRNA expression of costimulatory molecules and inflammatory cytokines. The data showed that Tmod1 $^{+/+}$and TOT/Tmod1 $1^{-/}$imDCs had low and comparable expression levels of MHC-II, CD80, CD40, and CCR7. However, the expression of CD86 was significantly lower in TOT/Tmod1 ${ }^{-1}$ imDCs compared to $T \bmod 1^{+++}$imDCs $(p<0.001$, Figure $3 C)$. LPS treatment induced the upregulation of all the maturation markers in the two genotypes. The expression levels of CD80 and CD86 were significantly lower in TOT/Tmod $1^{-/-}$mDCs than in Tmod $1^{+/+}$mDCs $(\mathrm{p}<0.05$, Figure 3C), while expression levels of $M H C-I I, C D 40$, and CCR7 were similar between the two groups. In terms of the mRNA expression of cytokines, we found that the expression of Il6 and Ifng in TOT/Tmod $1^{-/-}$mDCs was about $50 \%$ less than that in Tmod $1^{+/+}$mDCs $(\mathrm{p}<0.05$, Figure 3D), but the expression of Tnfa and Il12 was not significantly different between the two groups. These data suggest that Tmod1 deficiency has different and complex effects on the expression of costimulatory molecules and inflammatory cytokines.

\section{Tmod1 Deficiency Promotes the Activation of TLR4-TRIF-Dependent Pathway and Expression of Type I Interferons in DCs}

In addition to the MyD88-dependent pathway, the binding of LPS with TLR4 also triggers the activation of the TRIFdependent pathway, resulting in the phosphorylation of IRF3 and the expression of type I interferons $(46,47)$. We treated imDCs of the two genotypes with LPS for 2, 4, and $6 \mathrm{~h}$, and analyzed the phosphorylation of IRF3 using western blotting. The data showed that, following LPS treatment, IRF3 phosphorylation gradually increased and reached the highest level by $6 \mathrm{~h}$ in $\operatorname{Tmod}^{+/+}$DCs. However, in TOT/Tmod $1^{-/-}$DCs, the phosphorylation level of IRF3 was significantly higher than that in $\operatorname{Tmod1}^{+/+}$DCs at 2 and $4 \mathrm{~h}(\mathrm{p}<0.01)$ and remained high up to $6 \mathrm{~h}$ (Figures $4 \mathrm{~A}, \mathrm{~B}$ ). If $n b$ and $I l 10$ are the two major downstream genes of IRF3 (9). We performed qPCR to compare their expression levels in LPS-induced $T \bmod 1^{+/+}$and TOT/ Tmod $1^{-/-}$mDCs. We found that the expression of Ifnb and Il10 


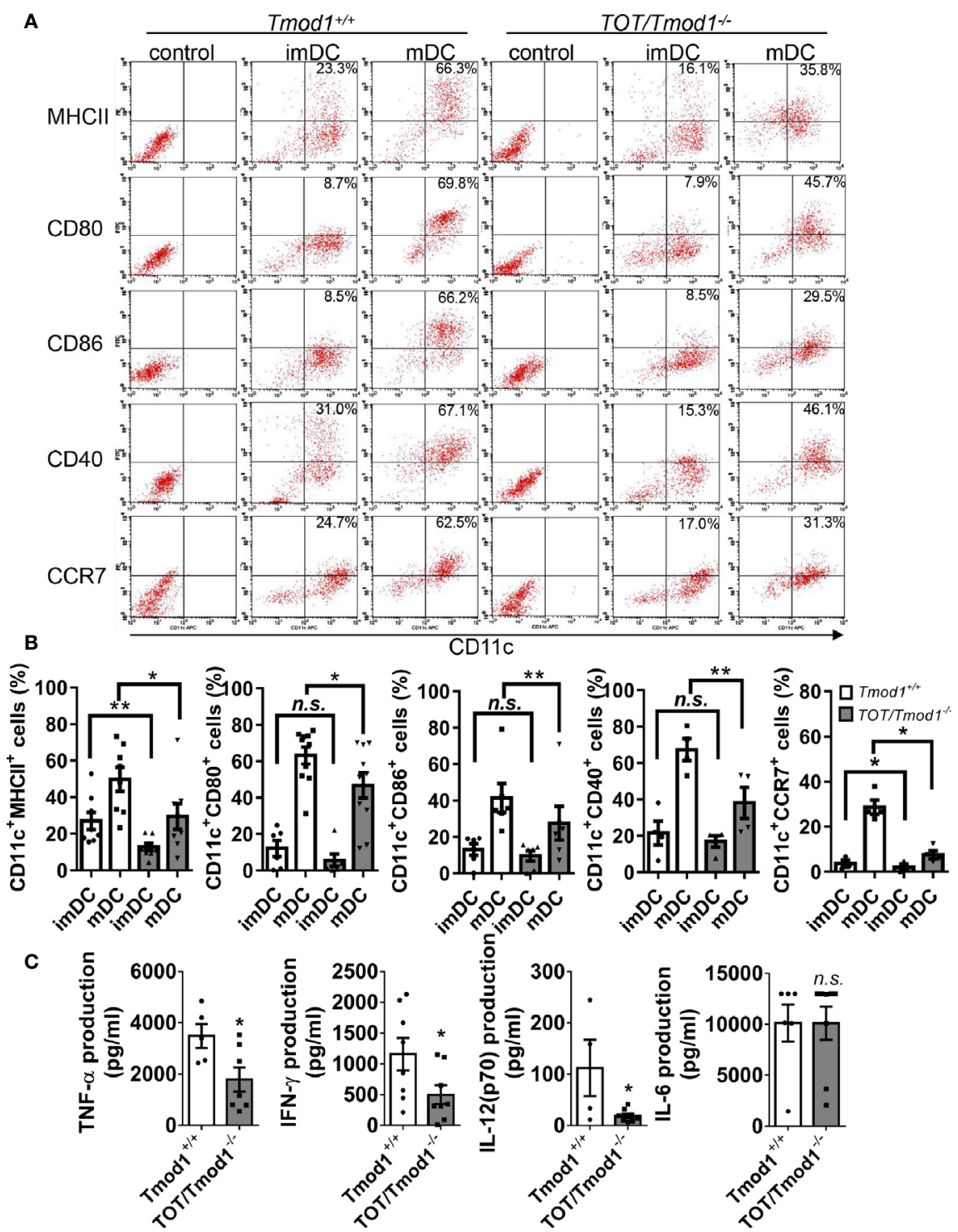

FIGURE 2 | Tmod1 is required for lipopolysaccharide (LPS)-induced costimulatory molecules and chemokine receptor expression and cytokine secretion in dendritic cells (DCs). (A) The immature DCs (imDCs) from Tmod1 ${ }^{+/+}$and TOT/Tmod1 $1^{-/}$mice were treated with 100 ng/ml LPS for 2 days, respectively, to induce maturation. Both imDCs and mature DCs (mDCs) of two genotypes were double-stained with APC-conjugated CD11c antibody and FITC- or PE-conjugated antibodies for MHC-II, CD80, CD86, CD40, and CCR7, and then analyzed with flow cytometry. imDCs of both genotypes were stained with isotype controls and run on flow cytometry to determine the gating. Representative dot plots were shown and the percentages of double-positive cells were labeled. (B) The statistical results for the percentages of $\mathrm{CD} 11 \mathrm{c}^{+} / \mathrm{MHC}-\mathrm{II}^{+}, \mathrm{CD} 11 \mathrm{c}^{+} / \mathrm{CD} 80^{+}, \mathrm{CD} 11 \mathrm{c}^{+} / \mathrm{CD} 86^{+}, \mathrm{CD} 11 \mathrm{c}^{+} / \mathrm{CD} 40^{+}$, and $\mathrm{CD} 11 \mathrm{c}^{+} / \mathrm{CCR} 7^{+}$cells for imDCs and $\mathrm{mDCs}$ from Tmod $1^{+/+}$and $\mathrm{TOT} /$ Tmod1 $^{-/-}$mice were shown. (C) ImDCs from Tmod $1^{+/+}$and TOT/Tmod $1^{-/-}$mice were induced to mature by treatment with 100 ng/ml LPS for 2 days and then culture media were collected. The concentrations of cytokines, TNF- $\alpha$, IFN- $\gamma$, IL-12(p70), and IL-6, in the culture media were measured on a flow cytometer by using a bead-based immunoassay kit. Data are presented as mean \pm SEM. ${ }^{\star} p<0.05,{ }^{\star \star} p<0.01$, n.s., no statistical significance; unpaired, two-tailed student's $t$-test.

was about 6- and 2- fold higher, respectively, in TOT/Tmod1 $1^{-/}$ mDCs compared to that in Tmod $1^{+/+}$mDCs (Figure 4C). Consistently, the concentrations of IFN- $\beta$ and IL-10 secreted in the culture medium of TOT/Tmod $1^{-/-}$mDCs were $318.8 \pm 13.8$ $\mathrm{pg} / \mathrm{ml}$ and $70.4 \pm 23.6 \mathrm{pg} / \mathrm{ml}$, respectively, which were markedly higher than those of Tmod1 $^{+/+}$mDCs $(280.6 \pm 10.5 \mathrm{pg} / \mathrm{ml}$ and $11.7 \pm 1.2 \mathrm{pg} / \mathrm{ml}$, respectively) (Figure 4D). These data suggest that Tmod1 deficiency promotes the activation of the TRIFdependent pathway and the expression of type I interferons.

Besides TLR4, other TLRs also signal via MyD88 or IRF or both, such as TLR2 agonist Pam3csk4 (MyD88-NF- $\mathrm{KB}$ pathway), TLR3 agonist poly (I:C) (TRIF-IRF3 pathway), and TLR9 agonist CpG ODN 1826 (MyD88-NF- $\kappa$ B and -IRF7 pathway) (48). To examine whether Tmod1 was required for signaling through 


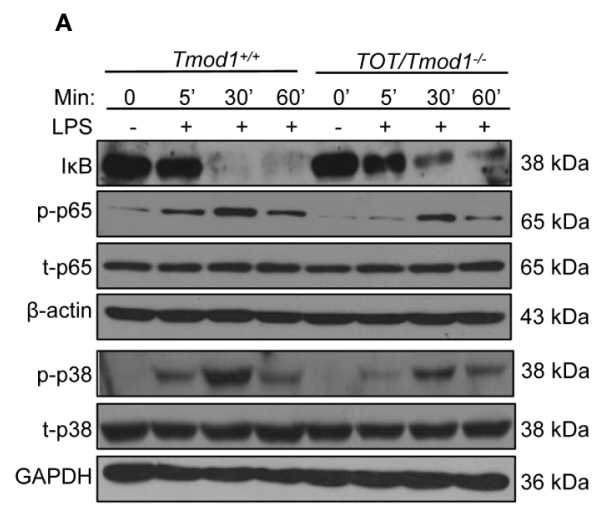

B
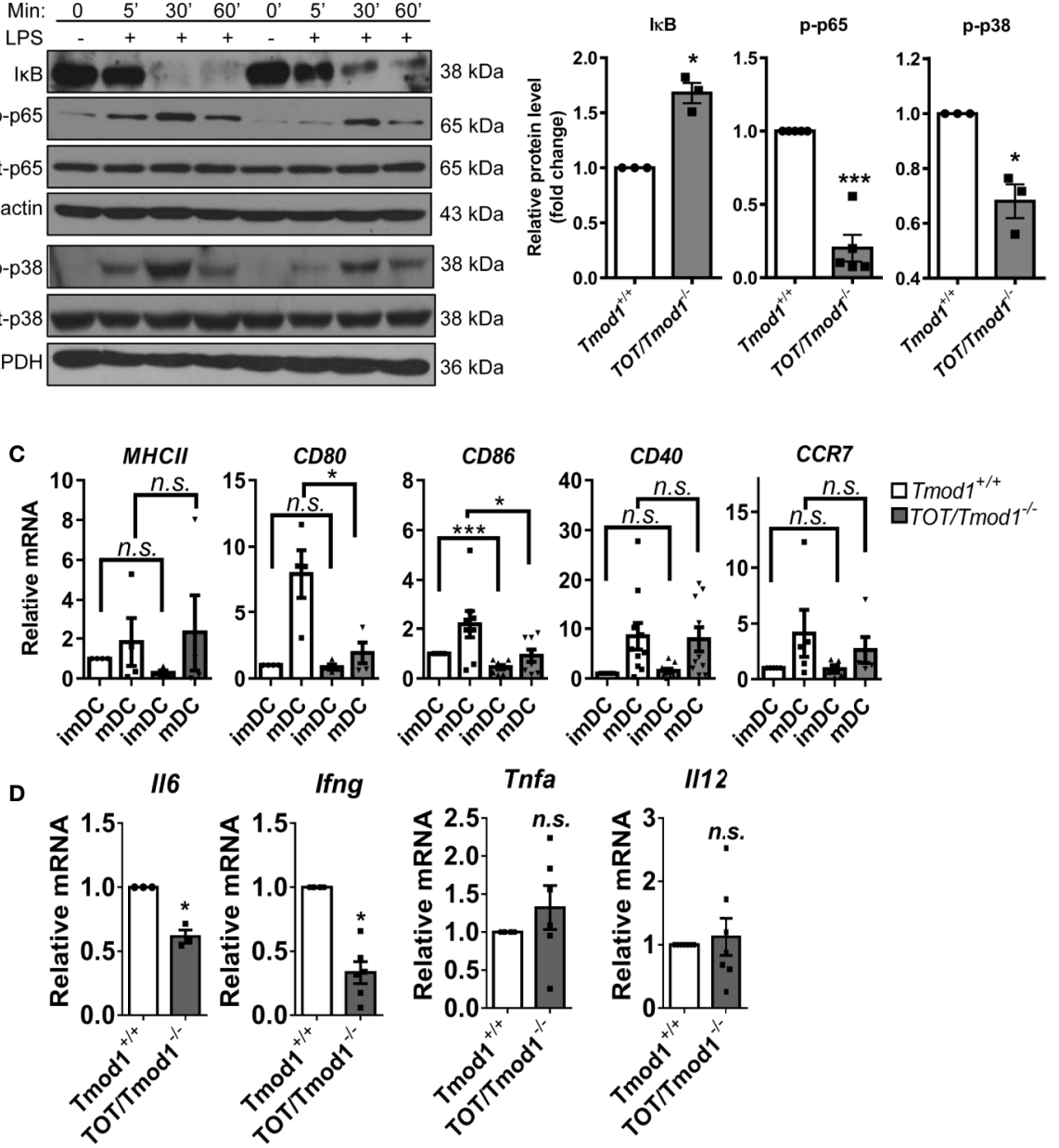

FIGURE 3 | Tmod1 regulates lipopolysaccharide (LPS)-induced phosphorylation of NF- $\mathrm{BB}$ and p38 MAPK and the expression of downstream costimulatory molecules and cytokines in DCs. (A) The immature DCs (imDCs) from Tmod1 ${ }^{+/+}$and TOT/Tmod $1^{-/}$mice were treated with LPS (100 ng/ml) for 0, 5, 30, $60 \mathrm{~min}$, respectively, and then total proteins were extracted. The expression of I $\mathrm{B}$, phosphorylated p65 (p-p65), total p65 (t-p65), phosphorylated p38 ( $\mathrm{p}$ p38), and total p38 (t-p38) were detected by western blotting by using specific antibodies. $\beta$-actin and GAPDH were used as internal controls. (B) The expressions of $1 \mathrm{kB}, \mathrm{p}-\mathrm{p} 65$, and $\mathrm{p}-\mathrm{p} 38$ at 5 min after LPS treatment were quantified after normalization to $\beta$-actin, $\mathrm{t}$-p65, or $\mathrm{t}$-p38. Their relative protein levels were presented as fold change. (C) The imDCs from Tmod $1^{+/+}$and TOT/Tmod $1^{-/}$mice were matured by LPS treatment for 2 days. Total RNAs were extracted from imDCs and mature DCs (mDCs) of both genotypes and qPCR was performed to detect the expression of $M H C l l, C D 80, C D 86, C D 40$, and CCR7. (D) The mRNA expression of cytokines, IL-6 (I/6), IFN- $\gamma$ (Ifng), TNF- $\alpha$ (Tnfa), and IL-12 (I/12), in LPS-induced mDCs from Tmod1 ${ }^{+/+}$and TOT/ Tmod $1 \%$ mice were detected by qPCR. Data are presented as mean \pm SEM. ${ }^{*} \mathrm{p}<0.05$, ${ }^{\star * *} \mathrm{p}<0.001$, n.s., no statistical significance; paired, two-tailed student's $t$-test.

other TLRs, we stimulated Tmod1 $1^{+/+}$and TOT/Tmod1 ${ }^{-/-}$imDCs with LPS, Pam 3 csk4, poly (I:C), and CpG. We observed a significant decrease in Il6 mRNA level and markedly augmentation in Illo and Ifnb mRNA levels by LPS in TOT/ Tmod1 $^{-/-}$imDCs (Figure S5A, C, D). But Pam3csk4, poly (I:C), and CpG failed to induce changes in Il6, Tnfa, Il10, and Ifnb mRNA expression in TOT/Tmod1 ${ }^{-/}$imDCs (Figure S5A-D). Consistently, TNF- $\alpha$ secretion was significantly inhibited and IL10 secretion was considerably enhanced by LPS in TOT/Tmod1 $1^{-/}$ imDCs, while other TLR agonists did not show much effect
(Figure S5E, F). These data suggest that Tmod1 may have a unique function on TLR4 signaling.

\section{Exogenous Tmod1 Expression Reversed the Effect of Tmod1 Deficiency on TLR4 Signaling}

Since Tmod1 deficiency resulted in inhibited TLR4/MyD88 signaling and enhanced TLR4/TRIF/IRF3 signaling, we next sought to explore whether exogenous Tmod1 could rescue these effects. We introduced exogenous Tmod1 into TOT/Tmod1 ${ }^{-/-}$ 

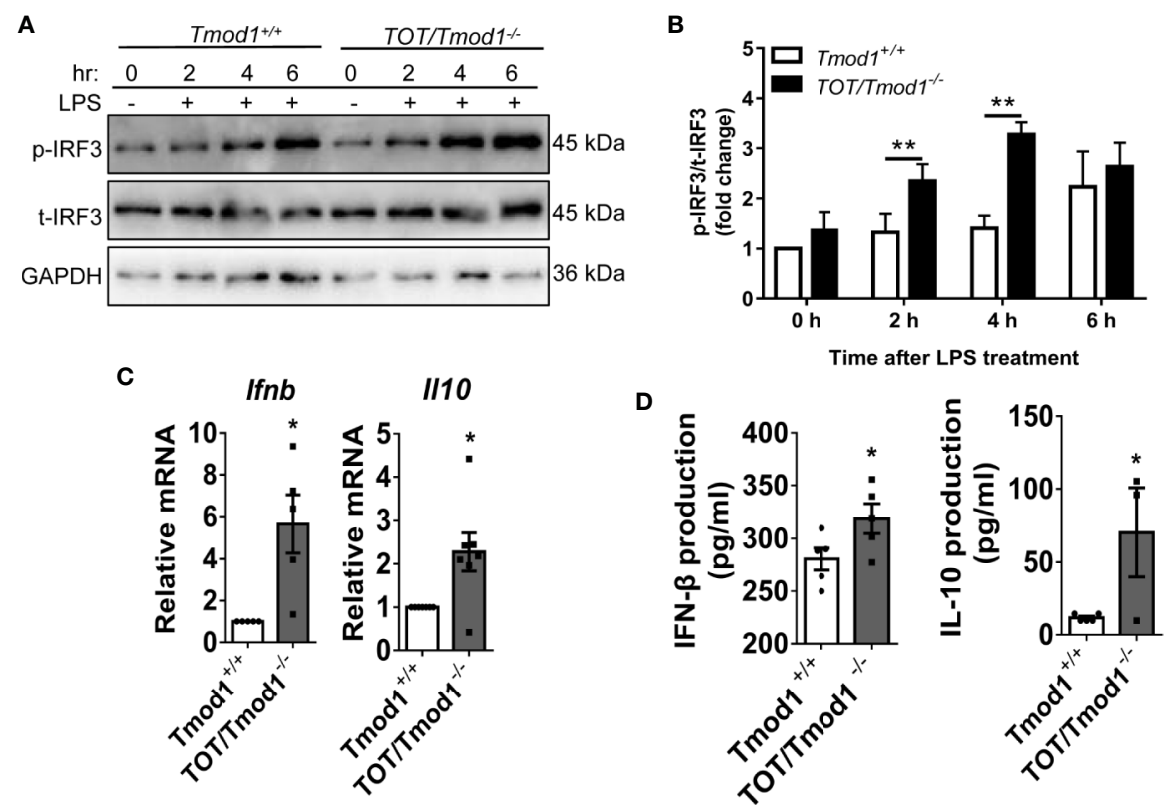

FIGURE 4 | Tmod1 deficiency promotes the activation of TRIF-dependent pathway and expression of type I interferons in dendritic cells (DCs). (A) The immature DC (imDCs) from Tmod $1^{+/+}$and TOT/Tmod $1^{-1-}$ mice were treated with LPS $(100 \mathrm{ng} / \mathrm{ml})$ for 0, 2, 4, $6 \mathrm{~h}$, respectively, and then total proteins were extracted. The expression of phosphorylated IRF3 (p-IRF3) and total IRF3 (t-IRF3) were detected by western blotting. GAPDH were used as an internal control. (B) The expressions of $\mathrm{p}$-IRF3 at different time points were quantified after normalization to t-IRF3 and the data was presented as fold change. (C) The imDCs from Tmod $1^{+/+}$and TOT/ $\mathrm{Tmod1}^{-1-}$ mice were matured by LPS treatment for 2 days. Total RNAs were extracted from imDCs and mature DCs (mDCs) of both genotypes and qPCR was performed to detect the expression of IFN- $\beta$ (Ifnh) and IL-10 (II10). (D) Culture media were collected from lipopolysaccharide (LPS)-induced mDCs from Tmod $1^{+/+}$ and TOT/Tmod $1^{-/}$mice. The concentrations of IFN- $\beta$ and IL-10 were measured on a flow cytometer by using a bead-based immunoassay kit. Data are presented as mean \pm SEM. ${ }^{*} \mathrm{p}<0.05,{ }^{* \star} \mathrm{p}<0.01$, paired, two-tailed student's $t$-test.

imDCs by adenovirus infection and then treated cells with LPS. The expressions of MyD88 and TRIF/IRF3 downstream genes were measured by qPCR and/or ELISA. qPCR data confirmed the reexpression of Tmod1 in Ad-Tmod1 infected cells (Figure 5A, left panel). Compared with Ad-Null infected mDCs, the expression of maturation marker, CD86, was increased to some extent in AdTmod1 infected mDCs (Figure 5A, second panel). Strikingly, the expressions of Il6, Tnfa, and Il12 were all significantly elevated, while the expression of $I l 10$ was greatly reduced ( $\mathrm{p}<0.05$, Figure $\mathbf{5 A}$, right panels). Furthermore, ELISA data showed that TNF- $\alpha$ secretion in Ad-Tmod1 infected mDCs was increased a little bit but did not show significance (Figure 5B). Yet, IL-10 secretion was significantly decreased ( $\mathrm{p}<0.05$, Figure 5C). These data suggest that rescue with exogenous Tmod1 expression in Tmod1-deficient DCs made MyD88-dependent pathway more activated and TRIFdependent pathway more inhibited, thus, it reversed the effect of Tmod1 deficiency on TLR4 signaling.

\section{Tmod1 Deficiency Did Not Affect the Endocytosis but Impaired the Migration Ability of DCs}

Endocytosis of antigens, migration, and antigen presenting abilities are the most important immune functions of DCs.
Therefore, we investigated whether Tmod1 deficiency affects these immune functions. ImDCs, residing in the peripheral tissues, engulf pathogens and antigens by endocytosis. We incubated $T \bmod 1^{+/+}$and TOT/Tmod1 $1^{-/}$imDCs with FITClabeled dextran for 0.5 and $2 \mathrm{~h}$ and detected their fluorescence intensities using flow cytometry. The data showed that the mean fluorescence intensities at $0.5 \mathrm{~h}$ were comparable between the two genotypes $(82.7 \pm 20.0$ vs. $69.5 \pm 13.5$, Figure 6A). The mean fluorescence intensities increased considerably at $2 \mathrm{~h}$, suggesting that more endocytosis occurred, but there was no difference between the two groups (Figure 6B). This indicates that Tmod1 deficiency did not affect the endocytosis ability of imDCs.

ImDCs patrol in the periphery by random migration, while mDCs undergo directional migration to lymph nodes because of high CCR7 expression $(49,50)$. Therefore, we used a modified Boyden chamber to measure the random migration of imDCs and mDCs, and chemotactic migration of mDCs in the two genotypes. We found that TOT/Tmod1 $1^{-/}$imDCs and mDCs showed significantly slower random migration compared to their Tmod $1^{+/+}$counterparts $(\mathrm{p}<0.01$ and $\mathrm{p}<0.05$, respectively, Figure 6C, left panel). When we measured the chemotaxis of mDCs toward CCL19, we found that the percentage of migrated TOT/Tmod1 ${ }^{-/}$ mDCs was half of that of migrated $T \bmod 1^{+/+}$mDCs (Figure 6C, 


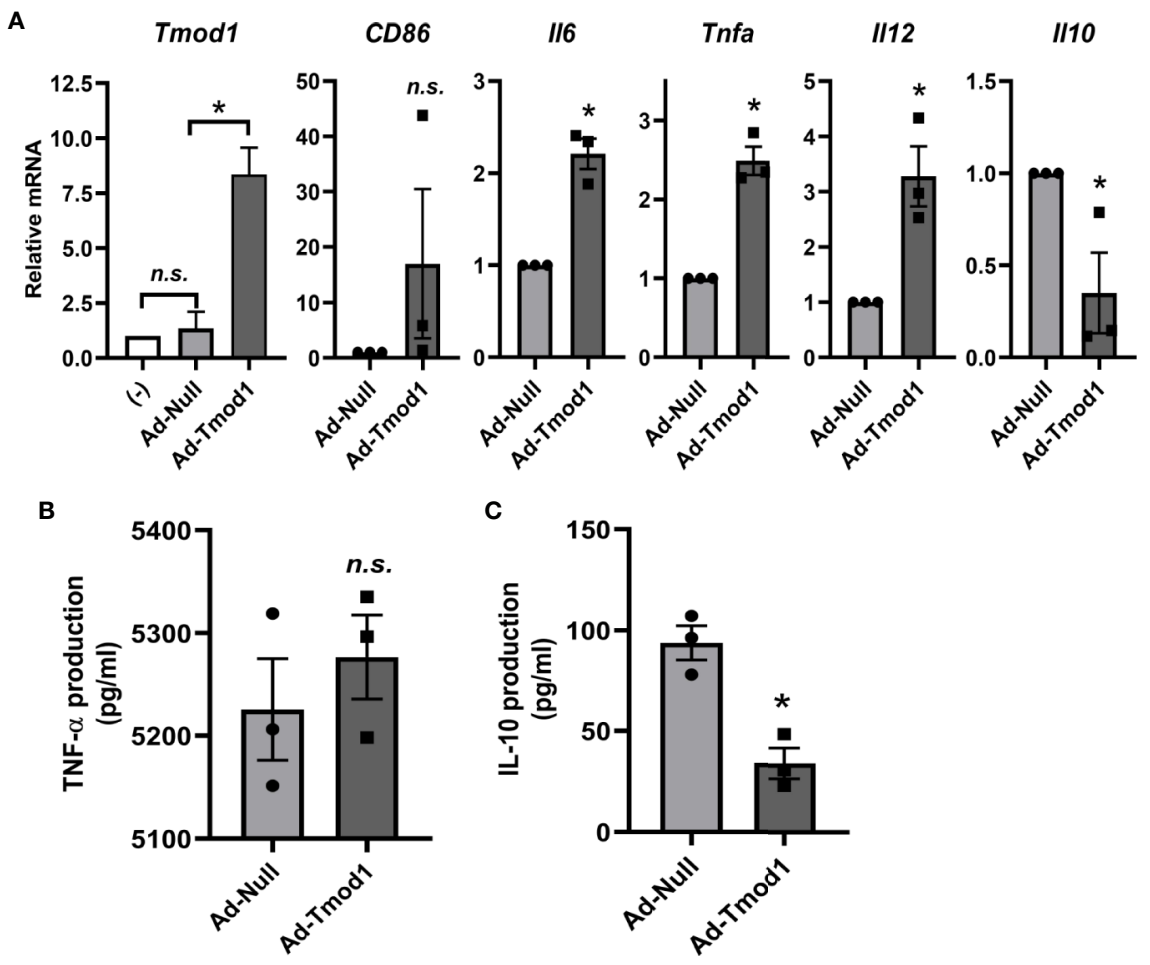

FIGURE 5 | Exogenous Tmod1 expression reversed the effect of Tmod1 deficiency on Toll-like receptor 4 (TLR4) signaling. (A) Immature dendritic cells (ImDCs) from TOT/Tmod1 ${ }^{-1}$ mice were infected with Tmod1 adenovirus (Ad-Tmod1) or control adenovirus (Ad-Null) at 200 multiplicity of infection (MOI) for 6 h. ImDCs without adenovirus treatment were used as the negative control (-). Adenovirus infected or un-infected imDCs were further treated with LPS (100 ng/ml) for $24 \mathrm{~h}$ and total RNAs were extracted. The mRNA expression of Tmod1, MyD88 downstream genes (CD86, I/6, Tnfa, and I/12) and TRIF/IRF3 downstream gene (I/10) were detected by qPCR. (B, C) Culture media were collected at the end of lipopolysaccharide (LPS) treatment. The concentrations of cytokines, TNF- $\alpha$ (B) and IL-10 (C), were measured by ELISA kits. Data are presented as mean \pm SEM. * $p<0.05$, n.s., no statistical significance; paired, two-tailed student's $t$-test.
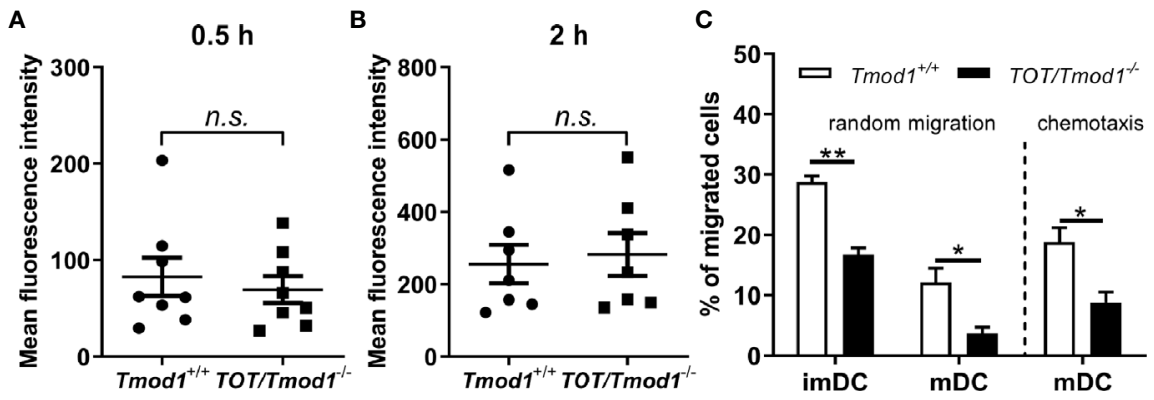

FIGURE 6 | Tmod1 deficiency did not affect the endocytosis but impaired the migration ability of dendritic cells (DCs). (A, B) Immature DCs (ImDCs) from Tmod1 ${ }^{+/+}$ and TOT/Tmod $1 \%$ mice were incubated with FITC-labeled dextran for $0.5 \mathrm{~h} \mathrm{(A)} \mathrm{and} 2 \mathrm{~h}(\mathbf{B})$, and their fluorescence intensities were analyzed by flow cytometry. (C) The imDCs and lipopolysaccharide (LPS)-induced mature DCs (mDCs) from Tmod $1^{+/+}$and TOT/Tmod1 ${ }^{1-}$ mice were plated in the upper compartment of the Boyden chamber and the culture medium for imDCs or mDCs was added to the lower compartment. After incubation for $12 \mathrm{~h}$, the numbers of randomly migrated cells were counted from the lower compartment. To measure the chemotaxis of mDCs, culture medium supplemented with CCL19 was placed in the lower compartment of Boyden chamber. The numbers of migrated mDCs were counted after 12 h. Data are presented as mean $\pm \mathrm{SEM}$. ${ }^{\star} \mathrm{p}<0.05$, ${ }^{\star \star} \mathrm{p}<0.01, n . s .$, no statistic significance; unpaired, two-tailed student's $t$-test. 
right panel). These data suggest that knockout of Tmod1 impaired the migration abilities of both imDCs and mDCs.

\section{Tmod1 Deficiency Impaired the T-Cell Stimulatory Ability of Mature DCs and Induced Immune Tolerance in the EAE Model}

$\mathrm{mDCs}$ are mainly responsible for presenting antigens to naïve $\mathrm{T}$ cells and priming them to produce inflammatory cytokines such as IFN- $\gamma$ and IL-10, and then mediating immune responses (15). Therefore, we sought to determine whether Tmod1 deficiency affects the T-cell stimulatory abilities of mDCs in vitro and in vivo. Allogeneic MLR data showed that $T \bmod 1^{+/+} \mathrm{mDCs}$ substantially promoted the proliferation of $\mathrm{T}$ cells, while the proliferation of $\mathrm{T}$ cells mixed with TOT/Tmod1 $1^{-/}$mDCs was significantly reduced $(\mathrm{p}<0.05$, Figure $7 \mathbf{A})$. To further validate this data, an in vivo assay was performed (41), in which C57BL/6J mice were transfused with OVA peptide-loaded $\operatorname{Tmod}^{+/+}$and
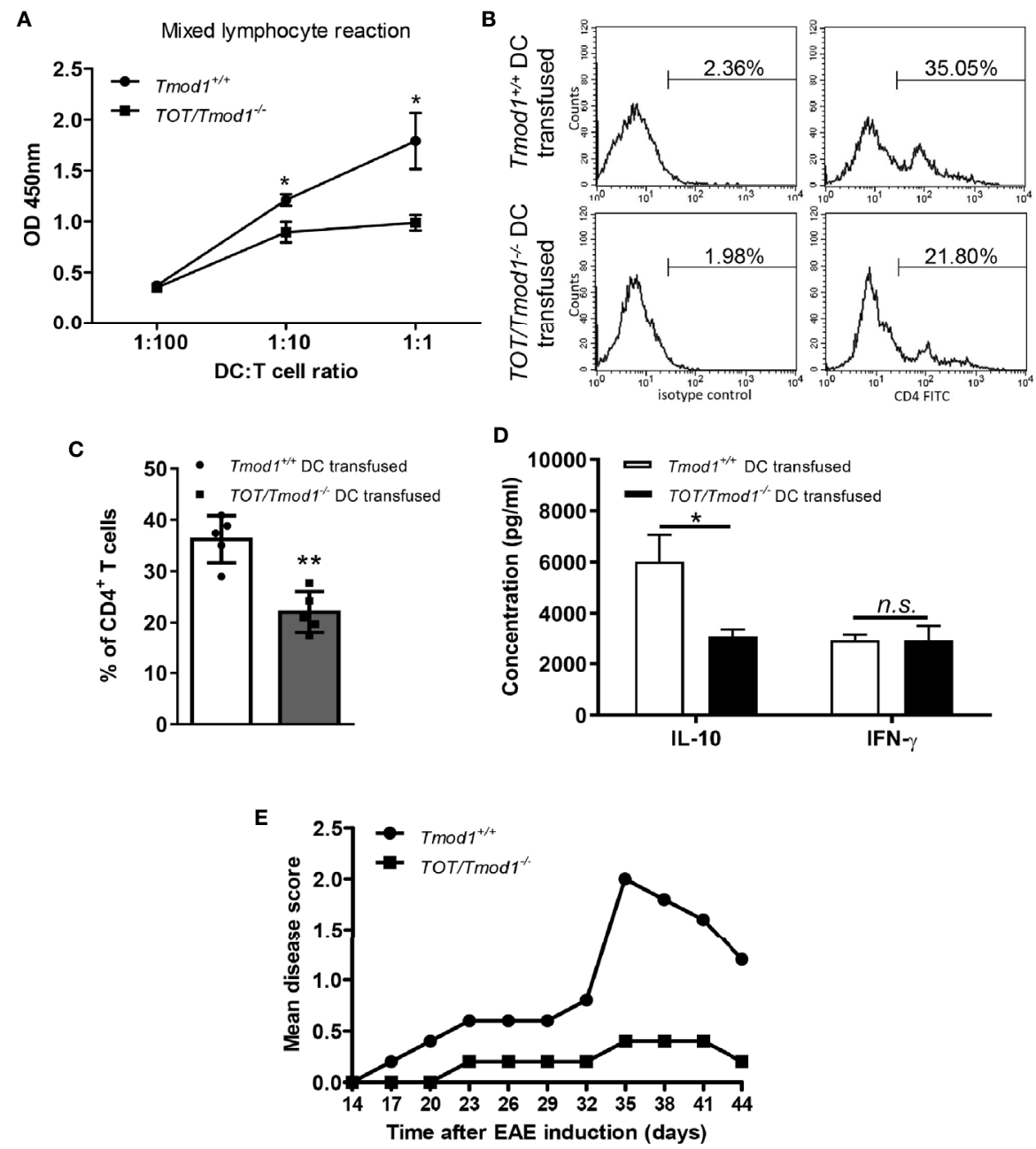

FIGURE 7 | Tmod1 deficiency impaired the T-cell stimulatory ability of mature dendritic cells (mDCs) and induced immune tolerance in the experimental autoimmune encephalomyelitis (EAE) model. (A) LPS-induced mDCs from Tmod $1^{+/+}$and TOT/Tmod $1^{-/-}$mice were incubated with $1 \times 10^{5} \mathrm{~T}$ cells in $1: 100,1: 10,1: 1$ ratios in the mixed lymphocytes reaction. The proliferation of T cells was measured by cell counting kit-8 and optical densities at $450 \mathrm{~nm}$ (OD450) were obtained.

(B, C) Lipopolysaccharide (LPS)-induced mDCs from Tmod $1^{+/+}$and TOT/Tmod1 ${ }^{-/}$mice (N=5) were treated with OVA peptide (323-339) and then transfused, respectively, to C57BL/6J mice at day 1, 3, and 5. On day 8, the spleen cells of transfused mice were collected and stimulated with OVA peptide (323-339) for another 3 days. The stimulated spleen cells were stained with FITC-conjugated anti-CD4 antibody or isotype control and analyzed by flow cytometry. The representative histograms were presented (B) and the percentages of CD4 ${ }^{+} T$ cells were obtained (C). (D) The culture media of OVA peptide-stimulated spleen cells were collected and the concentrations of IL-10 and IFN- $\gamma$ were measured on a flow cytometer by using a bead-based immunoassay kit. (E) LPS-induced mDCs from Tmod $1^{+/+}$and TOT/Tmod $1^{-/}$mice $(\mathrm{N}=5)$ were pulsed with MOG35-55 peptide and transfused, respectively, to C57BL/6J mice on day 0 and day 4. EAE was induced by injection of MOG35-55 peptide and Mycobacterium tuberculosis on day 7 and 14, and pertussis toxin on day 7 and 9 . The mice were observed and scored from day 14 until day 44 . The mean disease scores were plotted. Data are presented as mean \pm SEM. ${ }^{*} p<0.05$. ${ }^{\star \star} p<0.01$, n.s., no statistical significance; unpaired, two-tailed student's $t$-test. 
TOT/Tmod $1^{-/-}$mDCs, respectively, and their spleen cells were collected and re-stimulated with OVA peptide for 3 days. The spleen cells were stained with FITC-conjugated CD4 antibody and subjected to flow cytometry analysis. We found that the percentage of $\mathrm{CD}^{+} \mathrm{T}$ cells in the spleen cells from TOT/ Tmod1 $^{-1-}$ mDC transfused mice was $22.02 \pm 1.80 \%$, which was significantly lower than that in spleen cells from $\operatorname{Tmod}^{+/+} \mathrm{mDC}$ transfused mice $(36.23 \pm 2.05 \%)(p<0.01$, Figures $7 B, C)$. We also measured the concentrations of IFN- $\gamma$ and IL-10 in the culture medium of the spleen cells by flow cytometry. The data showed that the spleen cells from TOT/Tmod1 ${ }^{-/-} \mathrm{mDC}$ transfused mice produced markedly less IL-10 compared to those from Tmod1 $1^{+/+}$mDC transfused mice $(3062.2 \pm 311.1$ pg/ml vs. $6021.7 \pm 1032.7 \mathrm{pg} / \mathrm{ml}, \mathrm{p}<0.05)$ (Figure 7D). However, the production of IFN- $\gamma$ was similar between the two groups (Figure 7D). These data suggest that TOT/Tmod1 ${ }^{-/}$ mDCs were defective in $\mathrm{T}$ cell activation and priming. Moreover, Tmod1-deficient mDCs impaired the production of IL-10-producing $\mathrm{T}$ cells, but not IFN- $\gamma$-producing $\mathrm{T}$ cells. The results also indicate that Tmod1-deficient mDCs may be able to induce peripheral tolerance.

To test this hypothesis, we compared the abilities of $T m o d 1^{+/+}$ and TOT/Tmod1 $1^{-/-}$mDCs to prevent the development of EAE in mice, which was induced through subcutaneous injection of an encephalitogenic MOG peptide. The data showed that the mean disease score in mice injected with $\mathrm{Tmod}^{+/+} \mathrm{mDCs}$ increased gradually from day 14 to day 35 ( 0 to 2.0 ), and then dropped after day 35 . However, the mean disease score in mice injected with TOT/Tmod1 $1^{-/}$mDCs remained $<0.5$ from day 14 to day 44 (Figure 7E), indicating that three prior injections of MOG peptide-pulsed TOT/Tmod $1^{-/}$mDCs offered almost completely protection against the neuropathological symptoms associated with EAE. This suggests that Tmod1-deficient mDCs induced tolerance to the MOG peptides.

\section{Tmod1 Deficiency Diminished LPS- Induced F-Actin Formation and Cell Stiffening in DCs}

The immune functions of DCs rely on their cytoskeletal structure and biomechanical properties. It has been shown that F-actin is increased in LPS-stimulated DCs (21). Since Tmod1 regulates the polymerization of actin filaments, it may participate in LPSinduced F-actin remodeling. Therefore, we analyzed the F-actin content in imDCs and mDCs of Tmod1 $1^{+/+}$and TOT/Tmod1 $1^{-/-}$ mice using flow cytometry and fluorescence microscopy. As shown in Figures 8A, B, the F-actin content was significantly
A

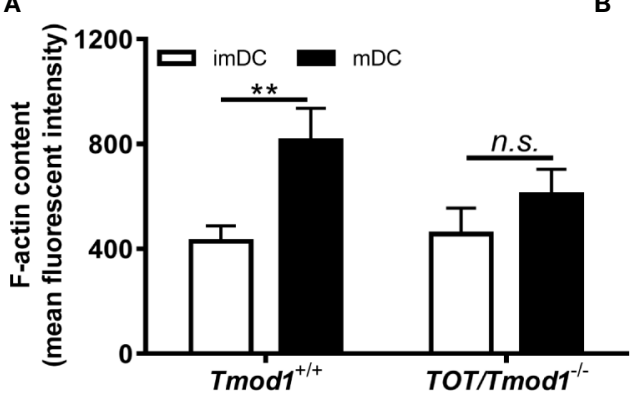

B

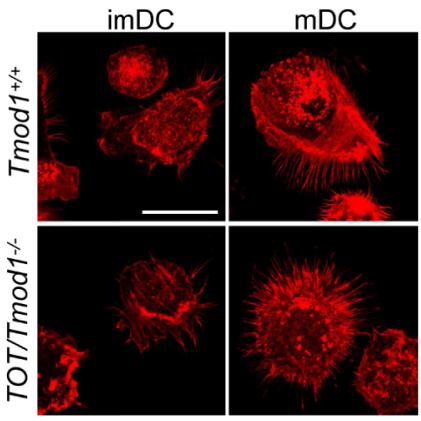

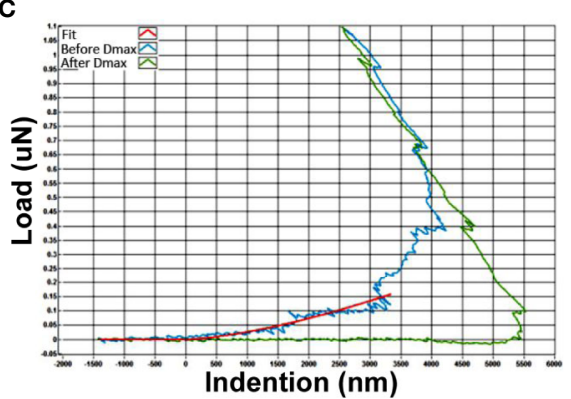

D

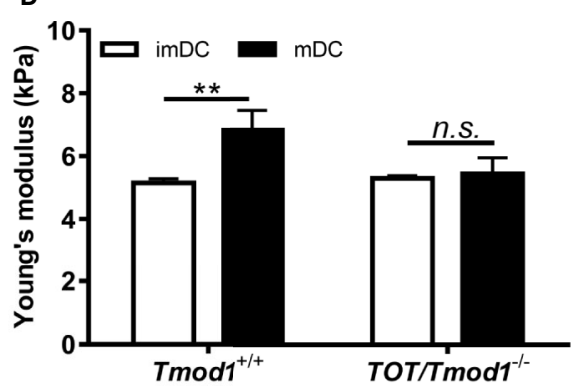

FIGURE 8 | Tmod1 deficiency diminished lipopolysaccharide (LPS) induced F-actin formation and cell stiffening in dendritic cells (DCs). (A) The immature DCs (imDCs) from Tmod $1^{+/+}$and TOT/Tmod1 $1^{-/}$mice were treated with LPS for 2 days to induce maturation. imDCs and mature DCs (mDCs) of both genotypes were stained with rhodamine phalloidin and subjected to flow cytometry analysis. The mean fluorescence intensities were obtained. (B) The imDCs and mDCs from Tmod $1^{+/+}$and TOT/Tmod $1^{-/-}$mice were cultured on poly L-Lysine-treated coverslips and stained with rhodamine phalloidin. The fluorescent signals were observed on the confocal microscopy. Representative images were shown. Bar $=25 \mu \mathrm{m}$. (C, D) The imDCs and mDCs of Tmod $1^{+/+}$and TOT/Tmod1 ${ }^{-/-}$mice were placed on a nanoindenter to measure the cell stiffness. The representative indention-load curve was shown (C). The curves were fitted using the Hertzian contact model and the Young's moduli of cells ( $\mathrm{N}=20 \sim 25)$ were calculated (D). Data are presented as mean $\pm \mathrm{SEM}$. ${ }^{* *} \mathrm{p}<0.01$, n.s., no statistical significance; unpaired, two-tailed student's t-test. 
increased in $T$ mod $1^{+/+}$mDCs compared to Tmod $1^{+/+}$imDCs $(\mathrm{p}<0.01)$. However, no difference was found in the F-actin contents of TOT/Tmod1 $1^{-/-}$imDCs and mDCs. We further measured the cell stiffness of imDCs and mDCs using a nanoindenter, in which the cells were indented with a probe and the loading (blue) and unloading (green) curves were drawn (Figure 8C). Young's modulus was calculated by fitting the curves with the Hertzian contact model. We found that Tmod $1^{+/+}$mDCs had greater Young's moduli than Tmod1 $1^{+/+}$ imDCs, while TOT/Tmod1 $1^{-/}$imDCs and mDCs had similar Young's moduli (Figure 8D). Our data suggest that Tmod1 deficiency diminished LPS-induced F-actin formation and cell stiffening in the DCs.

\section{DISCUSSION}

In the present study, we show evidence that Tmod1, an actincapping protein, is expressed in DCs and plays indispensable roles in controlling the maturation and multiple immune functions of DCs. Although Tmod1 was not essential for the differentiation of DCs, it was differentially expressed and had distinct functions in DC maturation. Tmod1 expression level was relatively low in imDCs (Figure 1). Tmod1 deficiency did not affect their endocytosis, but substantially impaired their free migration abilities (Figure 6), suggesting that Tmod1 has limited roles in the immune functions of imDCs. Tmod1 was upregulated in LPS-induced mDCs, which may be a result of the activation of the NF- $\mathrm{BB}$ signaling pathway (51). Importantly, our data showed that Tmod1 regulates the expression of costimulatory molecules and chemokine receptors (Figure 2), secretion of cytokines (Figure 2), migration abilities (Figure 6), and T-cell stimulatory abilities of mDCs (Figure 7).

The upregulation of MHC-II, costimulatory molecules, and chemokine receptors, and the secretion of inflammatory cytokines are the key features of DC maturation (7). The significantly low cell surface expression of MHC-II, CD80, CD86, CD40, and CCR7, and the decreased secretion of TNF$\alpha$, IL-12(p70), and IFN- $\gamma$ in TOT/Tmod $1^{-1-}$ mDCs suggests that TOT/Tmod1 $1^{-/-}$mDCs are less mature than $T$ mod1 $1^{++}$mDCs (Figure 2). This indicates that Tmod1 plays a critical role in regulating LPS-induced DC maturation. The underlying mechanisms may lie in the effects of Tmod1 on the TLR4mediated signaling pathways. Downstream of TLR4, the MyD88dependent NF- $\mathrm{KB}$ and MAPK pathways control the expression of costimulatory molecules and proinflammatory cytokines (5255). Western blotting data showed that compared to Tmod1 $1^{+/+}$ DCs, degradation of IKB was delayed and phosphorylation of p65 and p38 was reduced in TOT/Tmod1 $1^{-/-}$DCs under LPS stimulation (Figures 3A, B). This suggests that the NF- $\mathrm{BB}$ and p38 MAPK pathways were significantly inhibited when Tmod1 was deficient. Consistently, the gene expression levels of CD80, CD86, IL-6, and IFN- $\gamma$ were found to be decreased in LPS-treated TOT/Tmod1 ${ }^{-/}$DCs (Figures 3C, D). We noticed that although the gene expression of MHC-II, CD40, CCR7, IL12, and TNF- $\alpha$ did not change in the LPS-treated TOT/Tmod1 $1^{-/}$DCs, their surface expression or secretion were decreased (Figure 2). It has been shown that MHC-II is translocated onto the membrane when DCs mature (56). The secretion of inflammatory cytokines involves the trafficking of endosomal compartment (57). There is mounting evidence showing that the cytoskeleton can provide a scaffold for intracellular material transportation $(58,59)$. Therefore, Tmod1 may affect the trafficking of these membrane molecules and cytokines by regulating the actin cytoskeleton, rather than regulating their mRNA expression. This also indicates that Tmod1 has complex effects on the DC maturation process.

The TRIF/IRF3 pathway, activated when TLR4 is endocytosed into the endosomal compartments, promotes the expression of anti-inflammatory cytokines (9, 60, 61). The induction of IRF3 does not occur from plasma membranelocated TLR4 (62). Our data showed that after LPS treatment, phosphorylation of IRF3 was considerably higher in TOT/ Tmod1 $^{-/}$DCs than in Tmod1 ${ }^{+/+}$DCs (Figures 4A, B). The expression and secretion of anti-inflammatory cytokines, IFN- $\beta$ and IL-10, were also found to be significantly increased in TOT/ Tmod1 $^{-1-}$ DCs (Figures 4C, D). These results suggest that activation of the TRIF/IRF3 pathway was enhanced, and thus the endocytosis of TLR4 might be augmented when Tmod1 was deficient. It was shown that the release of IL-10, during DC maturation, could interfere with the upregulation of costimulatory molecules, CD80/86, and the production of proinflammatory cytokines, such as INF- $\gamma$, TNF- $\alpha$, and IL-12 (63-67). This autocrine effect of IL-10 may be a reason for TOT/ $\mathrm{Tmod1}^{-/-}$mDCs to display an immature phenotype. In addition, in TLR4 signaling, MyD88-dependent proinflammatory and TRIF-dependent anti-inflammatory pathways are competitive and thus restrict the activation of one another (68). Therefore, the enhanced activation of the TRIF/IRF3 pathway in Tmod1deficient DCs may negatively regulate the MyD88-dependent pathway. The question remains as to how Tmod1 regulates TLR4 endocytosis and TRIF/IRF3 pathway. Our preliminary data showed that Tmod1 deficiency did not affect the expression of TLR4 and MyD88 in DCs, but significantly upregulated the expression of CD14, which controls LPS-induced endocytosis of TLR4 (69, 70). Consistently, in macrophages overexpressing Tmod1, we observed the downregulated CD14 expression and less localization of TLR4 in early endosome and lysosome. However, further studies are needed to understand the precise mechanism through which Tmod1 regulates the expression of CD14.

It should be pointed out that the alteration of TLR4 signaling induced by Tmodl deficiency did not come from the compensatory effect of other Tmod homologs (Figure S2). Moreover, rescue with exogenous Tmod1 expression in TOT/ Tmod1 ${ }^{-/}$DCs reversed the effect of Tmod1 deficiency on TLR4 signaling (Figure 5), providing a strong support for the important role of Tmod1 in regulating TLR4 signaling. Another interesting finding is that Tmod1 deficiency seemed to have no obvious effect on other TLR signaling (Figure S3), such as TLR2, TLR3, and TLR9, which also signals via MyD88 or 
TRIF/IRF (48). Considering that only LPS but not other TLRs could induce upregulation of Tmod1 in DCs (Figure S1), we speculate that Tmod1 may have a unique function in TLR4 signaling, which needs to be further explored.

Upon maturation, DCs gain the ability of chemotactic migration, which enables them to migrate from the sites of infection to the secondary lymphoid organs (14). This process is coordinated by the CCR7 ligands, CCL19 and CCL21 (71). Our data showed that compared with $T$ mod $1^{+/+}$mDCs, TOT/ Tmod1 $^{-/-}$mDCs displayed impaired chemotaxis to CCL19 (Figure 6C). This could be well explained by the reduced expression level of CCR7 in TOT/Tmod1 $1^{-1-}$ mDCs (Figure 2). Further, like in imDCs, Tmod1 deficiency also resulted in impaired ability of random migration in the mDCs. This indicates that Tmod1 plays an important role in regulating both random migration and chemotaxis throughout the lifetime of DCs. It is known that DCs adopt the mesenchymal migration mode to move in the extracellular matrix, which includes three classic steps: spreading of the leading edge, adhesion to substrates, and retraction of the uropods (72-74). A simulation of the random and directed motion of DCs showed that in a strong chemotactic field, the DCs orient their filopodia to one pole, while in random migration, the filopodia are organized randomly (75). The places where filopodia appear are determined by spatially organized actin polymerization centers around the cell periphery. Tmod1 may participate in the filopodia formation by regulating actin polymerization centers. Indeed, in Tmod1-deficient mDCs, actin polymerization (i.e., Factin formation) and cell stiffening induced by LPS were abolished (Figure 8). Retraction of the uropod is mediated by stress fibers, which produce traction force promoting the cell to move forward (74). A recent study showed that Tmod1 is the key components of contractile stress fibers in non-muscle cells (30). Therefore, Tmod1 may also affect the stress fibers and uropod retraction during DC migration.

The mission of mDCs is to present an antigen peptide to naïve $\mathrm{T}$ cells to subsequently initiate immune responses (14). Full activation of $\mathrm{T}$ cells requires two signals, i.e., the antigens held in MHC complex and costimulatory molecules on DCs, and the effect of cytokines $(76,77)$. T-cell activation is the basis for its further proliferation and expansion. Both allogeneic MLR and in vivo assays showed that TOT/Tmod1 $1^{-/}$mDCs had impaired ability to promote the proliferation of $\mathrm{CD} 4^{+} \mathrm{T}$ cells (Figure 7), suggesting that they were incompetent in stimulating $\mathrm{T}$ cells. This defect should result from the low expression of MHC-II and costimulatory molecules, CD80/86 and CD40, on the membrane of TOT/Tmod $1^{-/-}$mDCs (Figures 2A, B). The reduced secretion of cytokines, such as TNF- $\alpha$, IFN- $\gamma$, and IL-12, in TOT/Tmod1 $1^{-/-}$ mDCs (Figure 2C) would further worsen this defect. The formation of immunological synapse between $\mathrm{T}$ cells and DCs involves rearrangement of the F-actin in the cell membrane into polymers (78-80). The reduction of F-actin content in TOT/ Tmod1 $^{-/-}$mDCs (Figures 8A, B) may make it difficult to form immune synapses with $\mathrm{T}$ cells. Moreover, the impaired chemotactic migration would also prevent TOT/Tmod1 $1^{-/-}$
mDCs from migrating to lymphoid tissues and thus decrease the possibility of DC and T-cell interaction in vivo. Therefore, Tmod1 deficiency in mDCs resulted in defective antigen presentation, inadequate $\mathrm{T}$-cell activation, and thus immune tolerance. This is well supported by the fact that TOT/Tmod1 $1^{-/}$ mDCs almost completely prevented the occurrence of neuropathological symptoms in the EAE model (Figure 7E).

Recent studies have shown that in addition to imDCs and mDCs, there is an intermediate population of DC maturation, called semi-mature DCs or tolerogenic DCs (DCreg) $(81,82)$. The main characteristics of this population are: 1) low or normal expression of cell surface markers, including MHC-II and costimulatory molecules; 2) increased secretion of antiinflammatory cytokines (such as IL-10, retinoic acid, and TGF$\beta$ ); 3) decreased production of proinflammatory cytokines (such as IL-12, IL-6, TNF- $\alpha$, and IFN- $\gamma$ ); and 4) mediating immune tolerance $(83,84)$. Careful observation revealed that LPS-induced TOT/Tmod1 $1^{-/}$mDCs possessed almost all the characteristics of semi-mature DCs. This indicates that Tmod1 is an important molecule necessary for DC maturation.

In conclusion, our study identified the important roles of Tmod1 in DC maturation and multiple aspects of the immune functions of DCs. Tmod1 may be a potential target for regulating DC functions in order to exaggerate or suppress the immune responses in the body, which would be beneficial to the immunotherapy for many diseases including cancer, transplant rejection, infection, and autoimmune diseases.

\section{DATA AVAILABILITY STATEMENT}

The original contributions presented in the study are included in the article/Supplementary Material. Further inquiries can be directed to the corresponding authors.

\section{ETHICS STATEMENT}

The animal study was reviewed and approved by The Ethics committee of Peking University Health Science Center.

\section{AUTHOR CONTRIBUTIONS}

$\mathrm{XL}$ and $\mathrm{XX}$ conducted the experiments, analyzed the results, and wrote the paper. XW, JZ, JL, and XG assisted with various experiments. LAS provided the knockout and rescued mouse lines and reviewed the paper. WY and ZZ conceptualized and supervised the project, analyzed the data, and wrote the paper. All authors contributed to the article and approved the submitted version. 


\section{FUNDING}

This work was funded by grants from the National Natural Science Foundation of China (31570938, 31771280, 31771014, 31660258, 11762006), the Science and Technology Innovative Talent Team of Guizhou Province (2015-4021), the High-level Innovative Talents Training Program of Guizhou Province "100" Level Talents (2016-5676), the Science and Technology Foundation of Guizhou Province (2018-1412), and the Natural Science Research Project of Education Department of Guizhou Province (YJSCXJH 2019-068).

\section{REFERENCES}

1. Palucka K, Banchereau J. Dendritic Cells: A Link between innate and adaptive immunity. J Clin Immunol (1999) 19:12-25. doi: 10.1023/A:1020558317162

2. Steinman RM. Decisions about dendritic cells: past, present, and future. Annu Rev Immunol (2012) 30:1-22. doi: 10.1146/annurev-immunol-100311102839

3. Sabado R, Balan S, Bhardwaj N. Dendritic cell-based immunotherapy. Cell Res (2017) 27:74-95. doi: 10.1038/cr.2016.157

4. Belderbos RA, Aerts JGJV, Vroman H. Enhancing dendritic cell therapy in solid tumors with immunomodulating conventional treatment. Mol Ther Oncolyt (2019) 13:67-81. doi: 10.1016/j.omto.2019.03.007

5. Gardner A, Pulido AM, Ruffell B. Dendritic cells and their role in immunotherapy. Front Immunol (2020) 11:924. doi: 10.3389/fimmu.2020.00924

6. Iberg CA, Hawiger D. Targeting dendritic cells with antigen-delivering antibodies for amelioration of autoimmunity in animal models of multiple sclerosis and other autoimmune Diseases. Antibodies (Basel) (2020) 9:E23. doi: 10.3390/antib9020023

7. Hopkins RA, Connolly JE. The specialized roles of immature and mature dendritic cells in antigen cross-presentation. Immunol Res (2012) 53:91-107. doi: $10.1007 / \mathrm{s} 12026-012-8300-\mathrm{z}$

8. Takeda K, Akira S. Toll-like receptors. Curr Protoc Immunol (2015) 109:14.12.1-14.12.10. doi: 10.1002/0471142735.im1412s109

9. Ullah MO, Sweet MJ, Mansell A, Kellie S, Kobe B. TRIF-dependent TLR signaling, its functions in host defense and inflammation, and its potential as a therapeutic target. J Leukoc Biol (2016) 100:27-45. doi: 10.1189/jlb.2RI1115$531 \mathrm{R}$

10. Rocha-Perugini V, Martínez Del Hoyo G, González-Granado JM, Ramírez-Huesca M, Zorita V, Rubinstein E, et al. CD9 regulates major histocompatibility complex class II trafficking in monocyte-derived dendritic Cells. Mol Cell Biol (2017) 37:e00202-17. doi: 10.1128/MCB.00202-17

11. Wang H, Xu L, Wu Z, Chen X. CCR7, CD80/86 and CD83 in Yellow Catfish (Pelteobagrus Fulvidraco): molecular characteristics and expression patterns with bacterial infection. Fish Shellfish Immunol (2020) 102:228-42. doi: $10.1016 /$ j.fsi.2020.04.026

12. Funk T, Fuchs AR, Altdörfer VS, Klein R, Autenrieth SE, Müller MR, et al. Monocyte-derived dendritic cells display a highly activated phenotype and altered function in patients with familial mediterranean fever. Clin Exp Immunol (2020) 201:1-11. doi: 10.1111/cei.13439

13. Hubo M, Trinschek B, Kryczanowsky F, Tuettenberg A, Steinbrink K, Jonuleit H. Costimulatory molecules on immunogenic versus tolerogenic human dendritic cells. Front Immunol (2013) 4:82. doi: 10.3389/fimmu.2013.00082

14. Worbs T, Hammerschmidt S, Förster R. Dendritic cell migration in health and disease. Nat Rev Immunol (2017) 17:30-48. doi: 10.1038/nri.2016.116

15. Hilligan KL, Ronchese F. Antigen presentation by dendritic cells and their instruction of CD4+ T helper cell responses. Cell Mol Immunol (2020) 17:58799. doi: 10.1038/s41423-020-0465-0

16. Vargas P, Maiuri P, Bretou M, Sáez PJ, Pierobon P, Maurin M, et al. Innate control of actin nucleation determines two distinct migration behaviours in dendritic cells. Nat Cell Biol (2016) 18:43-53. doi: 10.1038/ncb3284

17. Bendell AC, Anderson N, Blumenthal D, Williamson EK, Chen CS, Burkhardt JK, et al. Motile dendritic cells sense and respond to substrate geometry. Ann Biomed Eng (2018) 46:1348-61. doi: 10.1007/s10439-018-2041-7

\section{ACKNOWLEDGMENTS}

We would like to thank Editage (www.editage.com) for English language editing.

\section{SUPPLEMENTARY MATERIAL}

The Supplementary Material for this article can be found online at: https://www.frontiersin.org/articles/10.3389/fimmu.2020. 587441/full\#supplementary-material

18. Elizondo DM, Andargie TE, Haddock NL, Boddie TA, Lipscomb MW. Drebrin 1 in dendritic cells regulates phagocytosis and cell surface receptor expression through recycling for efficient antigen presentation. Immunology (2019) 156:136-46. doi: 10.1111/imm.13010

19. Malinova D, Fritzsche M, Nowosad CR, Armer H, Munro PM, Blundell MP, et al. WASp-dependent actin cytoskeleton stability at the dendritic cell immunological synapse is required for extensive, functional $\mathrm{T}$ cell contacts. J Leukoc Biol (2016) 99:699-710. doi: 10.1189/jlb.2A0215-050RR

20. Yamakita Y, Matsumura F, Lipscomb MW, Chou PC, Werlen G, Burkhardt JK, et al. Fascin 1 promotes cell migration of mature dendritic cells. J Immunol (2011) 186:2850-9. doi: 10.4049/jimmunol.1001667

21. Verdijk P, van Veelen PA, de Ru AH, Hensbergen PJ, Mizuno K, Koerten HK, et al. Morphological changes during dendritic cell maturation correlate with cofilin activation and translocation to the cell membrane. Eur J Immunol (2004) 34:156-64. doi: 10.1002/eji.200324241

22. Benvenuti F, Hugues S, Walmsley M, Ruf S, Fetler L, Popoff M, et al. Requirement of Rac1 and Rac2 expression by mature dendritic cells for $\mathrm{T}$ cell priming. Science (2004) 305:1150-3. doi: 10.1126/science.1099159

23. Schulz AM, Stutte S, Hogl S, Luckashenak N, Dudziak D, Leroy C, et al. Cdc42-dependent actin dynamics controls maturation and secretory activity of dendritic cells. J Cell Biol (2015) 211:553-67. doi: 10.1083/jcb.201503128

24. Oyarce C, Cruz-Gomez S, Galvez-Cancino F, Vargas P, Moreau HD, Diaz-Valdivia N, et al. Caveolin-1 expression increases upon maturation in dendritic cells and promotes their migration to lymph nodes thereby favoring the induction of CD8+ T cell responses. Front Immunol (2017) 8:1794. doi: $10.3389 /$ fimmu.2017.01794

25. Fowler VM. Identification and purification of a novel Mr 43,000 tropomyosinbinding protein from human erythrocyte membranes. J Biol Chem (1987) 262:12792-800.

26. Yamashiro S, Gokhin DS, Kimura S, Nowak RB, Fowler VM. Tropomodulins: Pointed-end capping proteins that regulate actin filament architecture in diverse cell types. Cytoskeleton (Hoboken) (2012) 69:337-70. doi: 10.1002/cm.21031

27. Yao W, Sung LA. Erythrocyte tropomodulin isoforms with and without the N-terminal actin-binding domain. J Biol Chem (2010) 285:31408-17. doi: $10.1074 /$ jbc.M110.130278

28. Yamashiro S, Speicher KD, Speicher DW, Fowler VM. Mammalian tropomodulins nucleate actin polymerization via their actin monomer binding and filament pointed end-capping activities. J Biol Chem (2010) 285:33265-80. doi: 10.1074/jbc.M110.144873

29. Kumari R, Jiu Y, Carman PJ, Tojkander S, Kogan K, Varjosalo M, et al. Tropomodulins Control the Balance Between Protrusive and Contractile Structures by Stabilizing Actin-Tropomyosin Filaments. Curr Biol (2020) 30:767-78.e5. doi: 10.1016/j.cub.2019.12.049

30. Gray KT, Stefen H, Ly TNA, Keller CJ, Colpan M, Wayman GA, et al. Tropomodulin's actin-binding abilities are required to modulate dendrite development. Front Mol Neurosci (2018) 11:357. doi: 10.3389/fnmol. 2018.00357

31. Chu X, Chen J, Reedy MC, Vera C, Sung KL, Sung LA. E-Tmod capping of actin filaments at the slow-growing end is required to establish mouse embryonic circulation. Am J Physiol Heart Circ Physiol (2003) 284:H1827H38. doi: 10.1152/ajpheart.00947.2002

32. Fritz-Six KL, Cox PR, Fischer RS, Xu B, Gregorio CC, Zoghbi HY, et al. Aberrant myofibril assembly in tropomodulin1 null mice leads to aborted 
heart development and embryonic lethality. J Cell Biol (2003) 163:1033-44. doi: $10.1083 /$ jcb.200308164

33. Yao W, Chu X, Sung LA. Cell type-restricted expression of erythrocyte tropomodulin Isoform41 in exon 1 knockout/LacZ knock-in heterozygous mice. Gene Expr Patterns (2015) 17:45-55. doi: 10.1016/j.gep.2015.02.001

34. Twine NC, Stover JA, Marshall B, Dukart G, Hidalgo M, Stadler W, et al. Diseaseassociated expression profiles in peripheral blood mononuclear cells from patients with advanced renal cell carcinoma. Cancer Res (2003) 63:6069-75.

35. Galligan CL, Baig E, Bykerk V, Keystone EC, Fish EN. Distinctive gene expression signatures in rheumatoid arthritis synovial tissue fibroblast cells correlates with disease activity. Genes Immun (2007) 8:480-91. doi: 10.1038/ sj.gene. 6364400

36. Labbé C, Boucher G, Foisy S, Alikashani A, Nkwimi H, David G, et al. Genome-wide expression profiling implicates a MAST3-regulated gene set in colonic mucosal inflammation of ulcerative colitis patients. Inflamm Bowel Dis (2012) 18:1072-80. doi: 10.1002/ibd.21887

37. Sussman MA, Welch S, Cambon N, Klevitsky R, Hewett TE, Price R, et al. Myofibril degeneration caused by tropomodulin overexpression leads to dilated cardiomyopathy in juvenile mice. J Clin Invest (1998) 101:51-61. doi: 10.1172/JCI1167

38. Green T, Vera C, Sussman MA, Martone M, Sung LA. Mechanobiology of erythrocytes from adult mice homozygous for a targeted disruption of the ETmod gene at exon 1. Cel Mol Bioeng (2011) 4:637-47. doi: 10.1007/s12195011-0203-x

39. Roney K. Bone marrow-derived dendritic dells. Methods Mol Biol (2019) 1960:57-62. doi: 10.1007/978-1-4939-9167-9_4

40. Zeng Z, Xu X, Zhang Y, Xing J, Long J, Gu L, et al. Tumor-derived factors impaired motility and immune functions of dendritic cells through derangement of biophysical characteristics and reorganization of cytoskeleton. Cell Motil Cytoskeleton (2007) 64:186-98. doi: 10.1002/ cm. 20175

41. Jiang A, Bloom O, Ono S, Cui W, Unternaehrer J, Jiang S, et al. Disruption of E-cadherin-mediated adhesion induces a functionally distinct pathway of dendritic cell maturation. Immunity (2007) 27:610-24. doi: 10.1016/ j.immuni.2007.08.015

42. Cox PR, Zoghbi HY. Sequencing, expression analysis, and mapping of three unique human tropomodulin genes and their mouse orthologs. Genomics (2000) 163:97-107. doi: 10.1006/geno.1999.6061

43. Cox PR, Fowler V, Xu B, Sweatt JD, Paylor R, Zoghbi HY. Mice lacking tropomodulin-2 show enhanced long-term potentiation, hyperactivity, and deficits in learning and memory. Mol Cell Neurosci (2003) 23:1-12. doi: 10.1016/S1044-7431(03)00025-3

44. Jan RH, Chen CJ, Hong YR, Lin YL, Chen LK. A surface antigen of orientia tsutsugamushi activates human monocyte-derived dendritic cells via nuclear factor-kB \& p38 mitogen-activated protein kinase pathways. Indian J Med Res (2018) 148:215-24. doi: 10.4103/ijmr.IJMR_1417_16

45. Moeller T, Wolfheimer S, Goretzki A, Scheurer S, Schülke S. NFkB- and MAP-Kinase signaling contribute to the activation of murine myeloid dendritic cells by a Flagellin A: allergen fusion protein. Cells (2019) 8:355. doi: 10.3390/cells 8040355

46. Csumita M, Csermely A, Horvath A, Nagy G, Monori F, Göczi L, et al. Specific enhancer selection by IRF3, IRF5 and IRF9 is determined by ISRE half-sites, 5 ' and 3' flanking bases, collaborating transcription factors and the chromatin environment in a combinatorial fashion. Nucleic Acids Res (2020) 4846:589604. doi: $10.1093 / \mathrm{nar} / \mathrm{gkz} 1112$

47. Aarreberg LD, Wilkins C, Ramos HJ, Green R, Davis MA, Chow K, et al. Interleukin- $1 \beta$ signaling in dendritic cells induces antiviral interferon responses. mBio (2018) 9:e00342-18. doi: 10.1128/mBio.00342-18

48. Mifsud EJ, Tan ACL, Jackson DC. TLR agonists as modulators of the innate immune response and their potential as agents against infectious disease. Front Immunol (2014) 5:79. doi: 10.3389/fimmu.2014.00079

49. Yoo JY, Jung NC, Lee JH, Choi SY, Choi HJ, Park SY, et al. Pdlim4 is essential for CCR7-JNK-mediated dendritic cell migration and F-actin-related dendrite formation. FASEB J (2019) 33:11035-44. doi: 10.1096/fj.201901031

50. Yashiro T, Takeuchi H, Nakamura S, Tanabe A, Hara M, Uchida K, et al. PU.1 plays a pivotal role in dendritic cell migration from the periphery to secondary lymphoid organs via regulating CCR7 expression. FASEB J (2019) 33:1148191. doi: 10.1096/fj.201900379RR
51. Ito-Kureha T, Koshikawa N, Yamamoto M, Semba K, Yamaguchi N, Yamamoto T, et al. Tropomodulin 1 expression driven by NF- $\kappa B$ enhances breast cancer growth. Cancer Res (2015) 75:62-72. doi: 10.1158/00085472.CAN-13-3455

52. Zhao B, Hui X, Jiao L, Bi L, Wang L, Huang P, et al. A TCM formula YYWY inhibits tumor growth in non-small cell lung cancer and enhances immuneresponse through facilitating the maturation of dendritic Cells. Front Pharmacol (2020) 11:798. doi: 10.3389/fphar.2020.00798

53. Liu S, Yang Y, Qu Y, Guo X, Yang X, Cui X, et al. Structural characterization of a novel polysaccharide from Panax notoginseng residue and its immunomodulatory activity on bone marrow dendritic cells. Int J Biol Macromol (2020) 161:797-809. doi: 10.1016/j.ijbiomac.2020.06.117

54. Jiang L, Huang D, Nie S, Xie M. Polysaccharide isolated from seeds of Plantago asiatica $\mathrm{L}$. induces maturation of dendritic cells through MAPK and NF-KB pathway. Saudi J Biol Sci (2018) 25:1202-7. doi: 10.1016/ j.sjbs.2017.09.011

55. Chuang CH, Cheng YC, Lin SC, Lehman CW, Wang SP, Chen DY, et al. Atractylodin suppresses dendritic cell maturation and ameliorates collageninduced arthritis in a mouse model. J Agric Food Chem (2019) 67:6773-84. doi: $10.1021 /$ acs.jafc.9b01163

56. Shin JS, Ebersold M, Pypaert M, Delamarre L, Hartley A, Mellman I. Surface expression of MHC class II in dendritic cells is controlled by regulated ubiquitination. Nature (2006) 444:115-8. doi: 10.1038/nature05261

57. Verboogen DRJ, Revelo NH, Ter Beest M, van den Bogaart G. Interleukin-6 secretion is limited by self-signaling in endosomes. J Mol Cell Biol (2019) 11:144-57. doi: 10.1093/jmcb/mjy038

58. Cross JA, Dodding MP. Motor-cargo adaptors at the organelle-cytoskeleton interface. Curr Opin Cell Biol (2019) 59:16-23. doi: 10.1016/j.ceb.2019.02.010

59. Hürtgen D, Vogel SK, Schwille P. Cytoskeletal and actin-based polymerization motors and their role in minimal cell design. Adv Biosyst (2019) 3:e1800311. doi: 10.1002/adbi.201800311

60. Tong W, Chen X, Song X, Chen Y, Jia R, Zou Y, et al. Resveratrol inhibits LPSinduced inflammation through suppressing the signaling cascades of TLR4-NFкB/MAPKs/IRF3. Exp Ther Med (2020) 19:1824-34. doi: 10.3892/etm.2019.8396

61. O'Loughlin T, Kruppa AJ, Ribeiro ALR, Edgar JR, Ghannam A, Smith AM, et al. OPTN recruitment to a Golgi-proximal compartment regulates immune signalling and cytokine secretion. J Cell Sci (2020) 133:jcs239822. doi: 10.1242/jcs.239822

62. Kagan JC, Su T, Horng T, Chow A, Akira S, Medzhitov R. TRAM couples endocytosis of Toll-like receptor 4 to the induction of interferon-beta. Nat Immunol (2008) 9(4):361-8. doi: 10.1038/ni1569

63. Aste-Amezaga M, Ma X, Sartori A, Trinchieri G. Molecular mechanisms of the induction of IL-12 and its inhibition by IL-10. J Immunol (1998) 160:5936-44.

64. Nizzoli G, Larghi P, Paroni M, Crosti MC, Moro M, Neddermann P, et al. IL10 promotes homeostatic proliferation of human $\mathrm{CD} 8(+)$ memory $\mathrm{T}$ cells and, when produced by CD1c (+) DCs, shapes naive CD8(+) T-cell priming. Eur J Immunol (2016) 46:1622-32. doi: 10.1002/eji.201546136

65. Corinti S, Albanesi C, la Sala A, Pastore S, Girolomoni G. Regulatory activity of autocrine IL-10 on dendritic cell functions. J Immunol (2001) 166:4312-8. doi: 10.4049/jimmunol.166.7.4312

66. De Smedt T, Van Mechelen M, De Becker G, Urbain J, Leo O, Moser M. Effect of interleukin-10 on dendritic cell maturation and function. Eur J Immunol (1997) 27:1229-35. doi: 10.1002/eji.1830270526

67. Sabat R, Grütz G, Warszawska K, Kirsch S, Witte E, Wolk K, et al. Biology of interleukin-10. Cytokine Growth Factor Rev (2010) 21:331-44. doi: 10.1016/ j.cytogfr.2010.09.002

68. Guven-Maiorov E, Keskin O, Gursoy A, VanWaes C, Chen Z, Tsai CJ, et al. The Architecture of the TIR Domain Signalosome in the Toll-like Receptor-4 Signaling Pathway. Sci Rep (2015) 5:13128. doi: 10.1038/srep13128

69. Wu Z, Zhang Z, Lei Z, Lei P. CD14: Biology and role in the pathogenesis of disease. Cytokine Growth Factor Rev (2019) 48:24-31. doi: 10.1016/ j.cytogfr.2019.06.003

70. Chen Z, Shao Z, Mei S, Yan Z, Ding X, Billiar T, et al. Sepsis Upregulates CD14 Expression in a MyD88-Dependent and Trif-Independent Pathway. Shock (2018) 49:82-9. doi: 10.1097/SHK.0000000000000913

71. Yan Y, Chen R, Wang X, Hu K, Huang L, Lu M, et al. CCL19 and CCR7 expression, signaling pathways, and adjuvant functions in viral infection and prevention. Front Cell Dev Biol (2019) 7:212. doi: 10.3389/fcell.2019.00212 
72. Cougoule C, Lastrucci C, Guiet R, Mascarau R, Meunier E, Lugo-Villarino G, et al. Podosomes, but not the maturation status, determine the proteasedependent 3D migration in human dendritic cells. Front Immunol (2018) 9:846. doi: 10.3389/fimmu.2018.00846

73. Sheetz MP, Felsenfeld D, Galbraith CG, Choquet D. Cell migration as a fivestep cycle. Biochem Soc Symp (1999) 65:233-43.

74. Lehtimäki J, Hakala M, Lappalainen P. Actin filament structures in migrating cells. Handb Exp Pharmacol (2017) 235:123-52. doi: 10.1007/164_2016_28

75. Parr A, Anderson NR, Hammer DA. A simulation of the random and directed motion of dendritic cells in chemokine fields. PloS Comput Biol (2019) 15: e1007295. doi: 10.1371/journal.pcbi.1007295

76. Saibil SD, Ohashi PS. Targeting T cell activation in immuno-oncology. Curr Oncol (2020) 27:S98-105. doi: 10.3747/co.27.5285

77. Corthay A. A three-cell model for activation of naïve T helper cells. Scand J Immunol (2006) 64:93-6. doi: 10.1111/j.1365-3083.2006.01782.x

78. Markey KA, Gartlan KH, Kuns RD, MacDonald KP, Hill GR. Imaging the immunological synapse between dendritic cells and T cells. J Immunol Methods (2015) 423:40-4. doi: 10.1016/j.jim.2015.04.029

79. Verboogen DR, Dingjan I, Revelo NH, Visser LJ, ter Beest M, van den Bogaart G. The dendritic cell side of the immunological synapse. Biomol Concepts (2016) 7:17-28. doi: 10.1515/bmc-2015-0028

80. Basu R, Huse M. Mechanical Communication at the Immunological Synapse. Trends Cell Biol (2017) 27:241-54. doi: 10.1016/j.tcb.2016.10.005
81. Lutz MB, Schuler G. Immature, semi-mature and fully mature dendritic cells: which signals induce tolerance or immunity? Trends Immunol (2002) 23:4459. doi: 10.1016/S1471-4906(02)02281-0

82. Devi KS, Anandasabapathy N. The origin of DCs and capacity for immunologic tolerance in central and peripheral tissues. Semin Immunopathol (2017) 39:137-52. doi: 10.1007/s00281-016-0602-0

83. Iberg CA, Jones A, Hawiger D. Dendritic cells as inducers of peripheral tolerance. Trends Immunol (2017) 38:793-804. doi: 10.1016/j.it.2017.07.007

84. Waisman A, Lukas D, Clausen BE, Yogev N. Dendritic cells as gatekeepers of tolerance. Semin Immunopathol (2017) 39:153-63. doi: 10.1007/s00281-016$0583-z$

Conflict of Interest: The authors declare that the research was conducted in the absence of any commercial or financial relationships that could be construed as a potential conflict of interest.

Copyright () 2021 Liu, Xia, Wang, Zhou, Sung, Long, Geng, Zeng and Yao. This is an open-access article distributed under the terms of the Creative Commons Attribution License (CC BY). The use, distribution or reproduction in other forums is permitted, provided the original author(s) and the copyright owner(s) are credited and that the original publication in this journal is cited, in accordance with accepted academic practice. No use, distribution or reproduction is permitted which does not comply with these terms. 